\title{
Swift-XRT follow-up of gravitational wave triggers during the third aLIGO/Virgo observing run
}

\author{
K.L. Page ${ }^{1}$, P.A. Evans ${ }^{1}$, A. Tohuvavohu ${ }^{2}$, J.A. Kennea ${ }^{3}$, N.J. Klingler ${ }^{3}$,
} S.B. Cenko ${ }^{4,5}$, S.R. Oates ${ }^{6}$, E. Ambrosi ${ }^{7}$, S.D. Barthelmy ${ }^{4}$, A.P. Beardmore ${ }^{1}$, M.G. Bernardini ${ }^{8}$, A.A. Breeveld ${ }^{9}$, P.J. Brown ${ }^{10}$, D.N. Burrows ${ }^{3}$, S. Campana ${ }^{8}$, R. Caputo ${ }^{4}$, G. Cusumano ${ }^{7}$, A. D'Aì ${ }^{7}$, P. D'Avanzo ${ }^{8}$, V. D'Elia ${ }^{11,12}$,

M. De Pasquale ${ }^{13}$, S.W.K. Emery ${ }^{9}$, P. Giommi ${ }^{12}$, C. Gronwall ${ }^{3,14}$, D.H. Hartmann ${ }^{15}$, H.A. Krimm ${ }^{16}$, N.P.M. Kuin ${ }^{9}$, D.B. Malesani ${ }^{17}$, F.E. Marshall ${ }^{4}$, A. Melandri ${ }^{8}$, J.A. Nousek ${ }^{3}$, P.T. O'Brien ${ }^{1}$, J.P. Osborne ${ }^{1}$, C. Pagani ${ }^{1}$, M.J. Page ${ }^{9}$, D.M. Palmer ${ }^{18}$, M. Perri ${ }^{12,11}$, J.L. Racusin ${ }^{4}$, T. Sakamoto ${ }^{19}$, B. Sbarufatti ${ }^{3}$, J.E. Schlieder ${ }^{4}$, M.H. Siegel ${ }^{3}$, G. Tagliaferri ${ }^{8}$ \& E. Troja ${ }^{4,20}$

1 School of Physics and Astronomy, University of Leicester, LE1 7RH, UK

2 Department of Astronomy and Astrophysics, University of Toronto, Toronto, ON, Canada

3 Department of Astronomy and Astrophysics, The Pennsylvania State University, University Park, PA 16802, USA

4 Astrophysics Science Division, NASA Goddard Space Flight Center, Greenbelt, MD 20771, USA

5 Joint Space-Science Institute, Computer and Space Sciences Building, University of Maryland, College Park, MD 20742, USA

6 School of Physics and Astronomy, University of Birmingham, B15 2TT, UK

7 INAF - IASF Palermo, Via Ugo La Malfa 153, I-90146, Palermo, Italy

8 INAF - Osservatorio Astronomico di Brera, Via Bianchi 46, I-23807 Merate, Italy

9 University College London, Mullard Space Science Laboratory, Holmbury St. Mary, Dorking, RH5 6NT, UK

10 George P. and Cynthia Woods Mitchell Institute for Fundamental Physics and Astronomy, Mitchell Physics Building, Texas A.EM. University, Department of Physics and Astronomy, College Station, TX 77843, USA

11 INAF-Osservatorio Astronomico di Roma, Via Frascati 33, I-00040 Monteporzio Catone, Italy

12 Space Science Data Center (SSDC) - Agenzia Spaziale Italiana (ASI), I-00133 Roma, Italy

13 Department of Astronomy and Space Sciences, Istanbul University, Beyazt 34119, Istanbul, Turkey

14 Institute for Gravitation and the Cosmos, The Pennsylvania State University, University Park, PA 16802, USA

15 Department of Physics and Astronomy, Clemson University, Kinard Lab of Physics, Clemson, SC 29634-0978, USA

16 National Science Foundation, Alexandria, VA 22314, USA

17 DTU Space, National Space Institute, Technical University of Denmark, Elektrovej 327, 2800 Kongens Lyngby, Denmark

18 Los Alamos National Laboratory, B244, Los Alamos, NM, 87545, USA

19 Department of Physics and Mathematics, Aoyama Gakuin University, Sagamihara, Kanagawa, 252-5258, Japan

20 Department of Physics and Astronomy, University of Maryland, College Park, MD 20742, USA

Accepted XXX. Received YYY; in original form ZZZ

\section{ABSTRACT}

The Neil Gehrels Swift Observatory followed up 18 gravitational wave (GW) triggers from the LIGO/Virgo/collaboration during the O3 observing run in 2019/2020, performing approximately 6500 pointings in total. Of these events, four where finally classified (if real) as binary black hole $(\mathrm{BH})$ triggers, six as binary neutron star (NS) events, two each of NSBH and Mass Gap triggers, one an unmodelled (Burst) trigger, and the remaining three were subsequently retracted. Thus far, four of these $\mathrm{O} 3$ triggers have been formally confirmed as real gravitational wave events. While no likely électromagnetic counterparts to any of these GW events have been identified in the X-ray data (to an average upper limit of $3.60 \times 10^{-12} \mathrm{erg} \mathrm{cm}^{-2} \mathrm{~s}^{-1}$ over $0.3-10 \mathrm{keV}$ ), or at other wavelengths, we present a summary of all the Swift-XRT observations performed during O3, together with typical upper limits for each trigger observed. The majority of X-ray sources detected during O3 were previously uncatalogued; while some of these will be new (transient) sources, others are simply too faint to have been detected by earlier survey missions such as ROSAT. The all-sky survey currently being performed by $e R O S I T A$ will be a very useful comparison for future observing runs, reducing the number of apparent candidate X-ray counterparts by up to 95 per cent.

Key words: gravitational waves - X-rays: general 


\section{INTRODUCTION}

Gravitational waves (GWs) were an important prediction of Einstein's 1915 General Theory of Relativity. Experiments to try and detect them were first pioneered back in the 1960s, with the first real steps towards LIGO (Laser Interferometer Gravitational-Wave Observatory) taken in the $1980 \mathrm{~s}^{1}$. LIGO was inaugurated in the final quarter of 1999, with science runs starting in 2002 (Abbott et al. 2004). Construction of the European Virgo project started in 1996, with the initial detector being completed in 2003. Science runs began in 2007, with a joint data analysis agreement with LIGO $^{1}$. The first follow-up of LIGO alerts by the Neil Gehrels Swift Observatory (Swift hereafter; Gehrels et al. 2004) occurred in 2010, though these two events were not astrophysical in origin (Evans et al. 2012).

'Advanced LIGO' (aLIGO; LIGO Scientific Collaboration 2015) began its first observing run (termed O1) on 2015 September 12, running until 2016 January 19, and yielding three triggers in that time. Of these, two (GW 150914 and GW 151226) were identified as binary black hole (BBH) mergers (Abbott et al. 2016a,b), with the third (G194575) being classified as a noise event. In addition, later offline analysis also suggested that trigger LVT 151012, while a lower significance detection, was still 87 per cent likely to be of astrophysical origin (Abbott et al. 2016c).

Following an upgrade and commissioning period, the second aLIGO observing run, O2, began on 2016 November 30, running until 2017 August 25. At the very end of this interval, from 2017 August 01, the Advanced Virgo detector (Acernese et al. 2015) also joined the run, allowing for three detector observations of GW events. During O2, a further eight confident GW triggers were identified (Abbott et al. 2019a) - seven BBHs, and one binary neutron star (BNS) merger, the latter leading to the first detection of an electromagnetic (EM) counterpart of a GW event (e.g., Abbott et al. 2017; Coulter et al. 2017; Evans et al. 2017; Goldstein et al. 2017; Hallinan et al. 2017; Pian et al. 2017; Troja et al. 2017).

The first part of the third observing run, O3a, ran from 2019 April 01 until 2019 September 30, at which time a one month commissioning break took place. O3b commenced on 2019 November 01, with the initial plan to run until 2020 April 30. However, due to the COVID-19 pandemic, the observing run was ended early, on March 27. O3 was the first observing run where triggers were publicly announced in real time, with details available online at https://gracedb.ligo.org/superevents/public/O3/. KAGRA (Kamioka Gravitational Wave Detector; KAGRA Collaboration 2019), Japan's GW observatory, began real-time observations in 2020 March, although the interferometer was not sensitive enough during the final weeks of $\mathrm{O} 3$ to detect the same GW events as LIGO and Virgo.

The discovery of GWs has opened up a new window on the cosmos, allowing astronomers to investigate sources which emit little, or no, light (and would therefore be invisible tơ traditional telescopes), and to delve into some

1 See https://www.ligo.caltech.edu/page/timeline for a brief history of LIGO. of the most extreme environments conceivable - the merging of $\mathrm{BH}$ and/or NS. While the initial detection of these events requires large GW interferometers, it is the subsequent follow-up by other observatories which can lead to good localisations, and details about temporal and spectral evolution of any afterglow. With its rapid response capability (typical slew times of $\lesssim 100 \mathrm{~s}$ ), together with co-aligned $\mathrm{X}$-ray and UV/optical telescopes, Swift is well placed to add to these observations. While there are many transients in the Universe, most of which will be unrelated to GW events (see discussion in Section 4.1), there are far fewer serendipitously detected in X-rays than at optical wavelengths. Searches for optical counterparts to O3 triggers have been published by Gompertz et al. (2020), Antier et al. (2020) and Kasliwal et al. (2020b), among others. In addition, a decaying X-ray afterglow is a distinguishing feature of many Gamma-Ray Bursts afterglows (GRBs; e.g., Nousek et al. 2006), with short GRBs expected to be formed during NS merger events, alongside the gravitational waves (Eichler et al. 1989; Mochkovitch et al. 1993). The $\mathrm{X}$-ray bandpass is therefore a useful and interesting region to search.

Evans et al. (2016c) summarised the Swift follow-up of LIGO triggers in O1, while Klingler et al. (2019) presented the same for O2. In this paper, we cover the Swift X-ray observations from O3. A companion paper by Oates et al. (in prep.) will present the corresponding optical and ultraviolet (UV) data from Swift, and a paper combining the $\gamma$-ray data from Swift and Fermi is also planned. We refer the reader to Evans et al. (2016c) and Klingler et al. (2019) for details of the X-ray data processing, analysis and source detection (see also Evans et al. 2014, 2020g), which did not significantly change for $\mathrm{O} 3$. Throughout the paper, upper limits are given at the $3 \sigma$ level. Magnitudes are provided in the AB system.

\section{SWIFT OBSERVATIONS}

The Swift observatory, launched in 2004, comprises three instruments: the wide-field ( $\sim 2$ sr) Burst Alert Telescope (BAT; Barthelmy et al. 2005), sensitive to $15-350 \mathrm{keV}$; the X-ray Telescope [XRT, $0.3-10 \mathrm{keV}$, with a circular field of view (FOV) of diameter 23.6 arcmin; Burrows et al. 2005]; and the UV/Optical Telescope (UVOT, with seven filters spanning 1700-6000 $\AA$, and a square FOV 17 arcmin each side; Roming et al. 2005).

Swift was designed to detect and rapidly follow up GRBs. While excellent at this job, its remit has expanded over the years, with the satellite becoming the go-to mission for any X-ray or UV transient source, especially where rapid observations are required; since 2015 , this has included the search for EM counterparts to GW events. The best case scenario would obviously be for Swift-BAT to trigger independently on a short GRB corresponding to a GW trigger, allowing the observatory to localise the source to a few arcmin (from BAT) or (sub)arcsec (UVOT/XRT) promptly and autonomously. Unfortunately, such a situation will be rare, and has not yet occurred; while GRB 170817A, the short burst associated with GW 170817, triggered the Fermi Gamma-ray Burst Monitor (GBM; Connaughton et al. 2017; Goldstein et al. 2017) and was detected by INTEGRAL (Savchenko et al. 2017), the location of the event 
was occulted by the Earth for Swift (Evans et al. 2017). Therefore, since the error regions of the LIGO GW detections are typically hundreds of square degrees in area, very much larger than the fields of view of the XRT or UVOT, a method to optimise Swift's ability to tile large areas of the sky was put in place (Evans et al. 2016a).

As mentioned by Evans et al. (2016b,c), the planned large-scale rapid tiling ability had not been commissioned by the start of O1. On 2016 January 13 an initial test of this rapid tiling was scheduled to observe GW 151226 (Evans et al. 2016c), showing that the spacecraft could safely perform hundreds of short $(\sim 60 \mathrm{~s})$ exposures in quick succession. This new observing mode, fully operational by the time of of O2, allows Swift to cover $\sim 50 \mathrm{deg}^{2}$ per day, a substantial increase over the possible response in O1. By the start of O3, work had been undertaken to optimise the scheduling of tiles for both efficiency and spacecraft safety (see discussion in Tohuvavohu 2018; Tohuvavohu \& Kennea 2018). In addition, further progress had been made such that these tiling observing plans could be uploaded more easily, allowing detailed follow-up by Swift to begin even more quickly.

Between each of the LIGO observing runs, the plan for Swift follow-up was optimised, based on lessons learned from the previous data. LIGO performs searches for two different types of event: Compact Binary Coalescence (CBC) and unmodelled (Burst) triggers ${ }^{2}$. The CBC triggers are modelled searches, looking specifically for BNS, NSBH and BBH mergers, while the unmodelled triggers have no prior assumptions regarding the signal, and could be caused by different astrophysical events (for example core-collapse supernovae).

For Swift observations of all CBC triggers, the $\mathrm{LVC}^{3}$ (LIGO Scientific Collaboration and Virgo Collaboration) probability map was convolved with an appropriate 3D galaxy catalogue (that is, the predicted distance information of the merger from Earth was included in the calculation), to account for the fact that such CBC events (the majority of LIGO triggers) are expected to occur in nearby galaxies. Further details of this process are given in Evans et al. (2016b) and Klingler et al. (2019). For all O3 CBC triggers, the Two Micron All Sky Survey (2MASS) Photometric Redshift Catalog (2MPZ; Bilicki et al. 2014) was used, For unmodelled triggers, a frequency of $>1 \mathrm{kHz}$ could correspond to events in our own Galaxy; in those specific cases, convolution with the Galactic plane was performed instead. For the lower frequency unmodelled events, the Gravitational Wave Galaxy Catalog (GWGC; White, Daw \& Dhillon 2011) was implemented, since these events are expected only to be detectable out to $\sim 100 \mathrm{Mpc}$ (Abbott et al. 2019b), and the GWGC data are more complete than $2 \mathrm{MPZ}$ in this regime.

In previous observing runs, the tiling pattern performed by Swift has been based entirely around the XRT FOV, since it is a little larger than the UVOT field (see fig. 3 of Evans et al. 2016c). While this avoids time wasted in overlapping sky area, it also leads to there being areas observed

https://emfollow.docs.ligo.org/userguide/analysis/searches.html 3 Throughout this paper, LVC is used as an abbreviation for publications by the LIGO Scientific Collaboration and the Virgo Collaboration by XRT, but outside the UVOT FOV. Given that the EM counterpart to GW 170817 was detected by the UVOT, not XRT (Evans et al. 2017), it was concluded that the tile selection criteria should be modified such that fields containing potential host galaxies can be offset or split, ensuring that galaxies fall entirely within the UVOT FOV (see also Klingler et al. 2019).

Klingler et al. (2019) details the follow-up criteria applied to triggers in $\mathrm{O} 2$ for the Swift observations. One change implemented at the start of O3a was that, for a BBH or Mass $\mathrm{Gap}^{4}$ trigger, we required that the minimum area enclosing 90 per cent of the probability in the convolved sky map be $\lesssim 10 \mathrm{deg}^{2}$. Previously the constraint had depended on fraction of the LVC probability region contained within the 400 most probable XRT fields. That is, given that the likelihood of the merger of two black holes leading to an EM counterpart is low (Kamble \& Kaplan 2013; Metzger 2019), it was decided that Swift would only follow up well-localised BBH events. One exception was made for S190414m, when a new version of the tiling software was tested; for this source, the area enclosing 90 per cent of the probability was $151.7 \mathrm{deg}^{2}$.

Initially, for O3a and earlier runs, any trigger which was marked as containing at least one NS (BNS or NSBH) was automatically flagged to be followed up, given that these are rarer than $\mathrm{BBH}$ mergers, and, from theory, more likely to lead to EM emission, in the form of a short GRB (if viewed on-axis; e.g., Berger 2014), and/or a kilonova, irrespective of jet alignment (since such emission is more isotropic; Eichler et al. 1989; Li \& Paczyński 1998; Metzger et al. 2010). However, during the month-long break between O3a and O3b, it was decided that the likelihood of the NS being disrupted should be taken into account; if a NS were simply to be swallowed whole by the companion BH, then no EM radiation would be expected. This was estimated using the equation of probabilities:

$$
\begin{array}{r}
P_{\text {disrupt.NS }}=P_{\mathrm{NS}} \times\left(1-P_{\text {Terres. }}\right)-P_{\mathrm{NSBH}} \\
+P_{\text {remnant }} \times P_{\mathrm{NSBH}}
\end{array}
$$

and changes were then implemented in the Swift selection algorithm on 2019 December 12. Here, $\mathrm{P}_{\text {disrupt.Ns }}$ is the probability of the event containing a disrupted NS; $\mathrm{P}_{\mathrm{NS}}$ is the probability that at least one of the compact objects is a NS (if the source is real), while $\mathrm{P}_{\mathrm{NSBH}}$ is that of the trigger being a NSBH binary. $\mathrm{P}_{\text {remnant }}$ gives the probability that the system ejected a non-zero amount of NS material. $\mathrm{P}_{\text {Terres. }}$ signifies the probability of a trigger being of terrestrial origin - i.e. noise. For each trigger, $\mathrm{P}_{\mathrm{NS}}, \mathrm{P}_{\text {Terres. }}, \mathrm{P}_{\mathrm{NSBH}}$ and $\mathrm{P}_{\text {remnant }}$ are taken from the relevant GCN (Gamma-ray Coordinates Network) notice sent by the $\mathrm{LVC}^{5}$. Throughout O3b there was also an additional requirement that the False Alarm Rate (FAR) needed to be $\lesssim 3.17 \times 10^{-9} \mathrm{~Hz}$ (i.e. less frequent than one in ten years) if $\mathrm{P}_{\text {disrupt.NS }}<0.7$; where the probability of a disrupted NS was higher, the FAR estimate was ignored. The area constraint was also rephrased in terms of the probability observable in 24 hours, $\mathrm{P}_{24 \mathrm{hr}}$, rather

4 A Mass Gap trigger implies a system with at least one compact object whose mass is in the hypothetical 'mass gap' between NS and $\mathrm{BH}$, taken to be $3-5 \mathrm{M}_{\odot}$

5 https://emfollow.docs.ligo.org/userguide/content.html 
than the statement that the area enclosing 90 per cent of the probability be $\lesssim 10 \mathrm{deg}^{2}$. Table 1 summarises what was required for follow-up by Swift for each type of trigger during the O3 observing run. We do note, however, that, while we tried to follow this decision tree systematically, Swift operational constraints also needed to be taken into consideration.

Assuming the criteria were satisfied, the standard follow-up plan for Swift was dependent on the type of event announced; the default steps are summarised in Table 2, though the scheme was not always fully executed (see description of individual triggers in Section 3). As mentioned above, the mergers of stellar mass black holes (the $\mathrm{BBH}$ triggers) are not generally anticipated to lead to EM radiation, so a promptly detected short GRB is not expected (c.f. GW 150914; Connaughton et al. 2016, 2018; Greiner et al. 2016); 500 s tiling observations were still performed however. When the system was thought to include a disrupted NS, or the trigger was a low-frequency unmodelled event, the observations took a two pronged approach: first rapid short tiles, repeated if the area could be covered in $<1.5$ days, followed by deeper observations. The reasoning behind this is that prompt observations would look for the rapidly fading (on-axis) afterglow (with the repeat short observations possibly serving to catch a source turning on slightly more slowly), while later ones might detect a rising (off-axis) jet (see Evans et al. 2016a, for full details). While off-axis mergers are more likely, due to simple geometric effects, the time at which the corresponding afterglow would be detectable by the XRT depends strongly on the jet parameters and the density of the circumburst medium. Observations of $500 \mathrm{~s}$ from three to seven days post-trigger were concluded to be a sensible compromise, following work by Evans et al. (2016a). For unmodelled triggers with a frequency $>1 \mathrm{kHz}$, the region was to be observed for $80 \mathrm{~s}$ per tile continuously for four days. These observing strategies are somewhat different from O2 (Klingler et al. 2019), though were not changed between O3a and O3b. In addition to this underlying plan, follow-up observations of externallydetected sources (typically optical transients) announced via the GCN would also be performed where deemed to be of interest.

Sources detected in the XRT observations were automatically ranked, indicating how likely each one was to be the EM counterpart of the GW triggen Evans et al. (2016b) provides the detailed definitions ${ }^{6}$, with Rank 1 being a candidate afterglow ${ }^{7}$; Rank 2 being an interesting source/possible EM counterpart ${ }^{8}$; Rank 3 indicating an un-

\footnotetext{
${ }^{6}$ See also https://www.swift.ac.uk/LVC/docs.php\#classes

7 To be marked as an afterglow candidate, a source must be either uncatalogued and at least $5 \sigma$ above the $3 \sigma$ upper limit from RASS or 1SXPS/2SXPS, or a known X-ray source which is $5 \sigma$ above the catalogued flux; a power-law spectrum with $\Gamma=1.7$ and $\mathrm{N}_{\mathrm{H}}=3 \times 10^{20} \mathrm{~cm}^{-2}$ is always used for the conversion between the ROSAT and Swift bands. Additionally, the source in question must lie within $200 \mathrm{kpc}$ of a known galaxy (assuming it is at the distance of that galaxy). There is no requirement that the source be seen to be fading immediately.

8 To be classed as 'interesting', a source must be either uncatalogued and at least $3 \sigma$ above the $3 \sigma$ upper limit from RASS or 1SXPS/2SXPS, or fading; alternatively, it may be a known X-ray
}

catalogued, though faint ${ }^{9}$, source unlikely to be an afterglow; and Rank 4 corresponding to a known X-ray source not showing any unusual activity. To determine if a source was a known X-ray emitter, the full HEASARC X-ray Master Catalogue ${ }^{10}$ was consulted, as well as the 1SXPS/2SXPS (Evans et al. 2014, 2020g) catalogues. In addition, comparisons with any overlapping reference fields previously observed for the Swift Gravitational Wave Galaxy Survey (SGWGS; Klingler et al. 2019, Tohuvavohu in prep.) were performed. SGWGS is a pre-imaging survey of the $\sim 14,000$ most likely host galaxies for BNS mergers within $\sim 100 \mathrm{Mpc}$, with data collected in the X-ray and UVOT ( $u$ and $u v w 1$ filters) bands. When complete, $\sim 41$ per cent of the integrated luminosity within $100 \mathrm{Mpc}$ will have XRT/UVOT templates with exposure times of $\sim 1 \mathrm{ks}$ with which to compare future observations.

GCN counterpart notices were initially automatically sent out for all source detected. From 2019 April 30, notices were disabled for sources of Rank 3 or 4 which had a warning flag set (see below); from 2019 May 03, Rank 4 counterpart notices were no longer sent out whether or not there was a warning flag.

Each source was then checked by a member of the XRT team, to catch any spurious detections (caused, for example, by optical loading ${ }^{11}$, diffuse emission or unusually high background - all of which would raise a 'warning' flag); only these 'confirmed' sources are listed on the public webpage at https://www,swift.ac.uk/LVC/. In most cases, humanvetted summary GCN circulars were then sent out when observations had been completed and checked. We take this opportunity to remind users once again that the GCN notices sent out for every XRT detection are automatic and preliminary, and are aimed at informing the community as rapidly as possible of potential counterparts. Both the warning flags in the notice, and, especially, the list of confirmed sources on the website should be checked carefully before accepting the validity of the source.

Following the tiling and initial source detection, any Rank 1 or 2 sources of interest were reobserved with high priority to check on the flux level and any variability. The default plan ${ }^{6}$ called for all Rank 3 sources also to be reobserved once the tiling was completed. While these follow-up observations were sometimes performed (particularly if the source showed any sign of fading, even if at $<2 \sigma$ ), the large number of the Rank 3 sources, together with other higher priority non-GW-related Swift observations, meant that this was not always the case. Rank 4 (catalogued) sources were not reobserved.

Table 3 provides the details of the triggers from O3 which Swift followed up, including the number of X-ray sources detected, while Table A1 lists all the O3 events, giving reasons why Swift observations were not performed where relevant.

source which is $3 \sigma$ above the catalogued flux. It does not need to be near a known galaxy.

9 By faint, we mean below the $3 \sigma$ ROSAT All-Sky Survey (RASS)

limit at the source position.

10 https://heasarc.nasa.gov/W3Browse/all/xray.html

11 https://www.swift.ac.uk/analysis/xrt/optical_loading.php 
Table 1. Swift follow-up criteria during O3. $\mathrm{P}_{0.9}$ signifies 90 per cent of the probability in the galaxy-convolved skymap; $\mathrm{P}_{24 \mathrm{hr}}$ signifies the galaxy-convolved probability which Swift would be able to observe in 24 hours.

\begin{tabular}{|c|c|}
\hline Type of trigger & Criteria \\
\hline \multicolumn{2}{|l|}{ O3a } \\
\hline $\begin{array}{l}\text { CBC; BBH } \\
\text { CBC; Mass Gap } \\
\text { CBC; NSBH } \\
\text { CBC; BNS } \\
\text { Unmodelled (Burst); frequency }<1 \mathrm{kHz} \\
\text { Unmodelled (Burst); frequency }>1 \mathrm{kHz}\end{array}$ & $\begin{array}{c}\text { Follow if } \mathrm{P}_{0.9} \lesssim 10 \mathrm{deg}^{2} \\
\text { Observe if } \mathrm{P}_{\mathrm{NS}}>0 \text { or } \mathrm{P}_{0.9} \lesssim 10 \mathrm{deg}^{2} \\
\text { Observe all triggers with } \mathrm{P}_{\mathrm{NS}}>0 \\
\text { Observe all triggers with } \mathrm{P}_{\mathrm{NS}}>0 \\
\text { Follow if } \mathrm{P}_{24 \mathrm{hr}}>0.5 \& \mathrm{FAR}<1 / \mathrm{yr} \text {. } \\
\text { Follow all }\end{array}$ \\
\hline \multicolumn{2}{|l|}{ O3b } \\
\hline 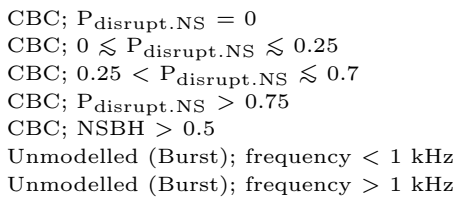 & $\begin{array}{l}\text { Follow if } \mathrm{P}_{24 \mathrm{hr}}>0.5 \& \mathrm{FAR}<1 / 10 \mathrm{yr} \text {. } \\
\text { Follow if } \mathrm{P}_{24 \mathrm{hr}}>0.5 \& \mathrm{FAR}<1 / 10 \mathrm{yr} \text {. } \\
\text { Follow if } \mathrm{P}_{24 \mathrm{hr}}>0.4 \\
\text { Follow if } \mathrm{P}_{24 \mathrm{hr}}>0.1 \\
\text { Follow if } \mathrm{P}_{24 \mathrm{hr}}>0.75^{*} \\
\text { Follow if } \mathrm{P}_{24 \mathrm{hr}}>0.5 \& \mathrm{FAR}<1 / \mathrm{yr} . \\
\text { Follow all }\end{array}$ \\
\hline
\end{tabular}

*This was an additional option included from 2019 November 07.

Table 2. Default tiling follow-up plan for Swift observations after a GW trigger.

\begin{tabular}{ll}
\hline Type of trigger & Steps \\
\hline$P_{\text {disrupt.NS }}=0$ & $500 \mathrm{~s}$ per field for 4 days, or until 90 per cent of the probability is covered, whichever sooner \\
\hline $\begin{array}{l}P_{\text {disrupt.NS }}>0 \\
\text { or } \\
\text { Unmodelled (Burst); frequency }<1 \mathrm{kHz}\end{array}$ & $\begin{array}{l}\text { (i) } 80 \mathrm{~s} \text { tiles up to } 800 \text { fields } * \text { or until } 90 \text { per cent of the probability had been covered, whichever sooner } \\
\text { (iii) } 500 \mathrm{~s} \text { tiling completed in }<1.5 \mathrm{~d} \text {, repeat until } \mathrm{T}+3 \mathrm{~d}\end{array}$ \\
\hline Unmodelled (Burst); frequency $>1 \mathrm{kHz}$ & $80 \mathrm{~s}$ per tile for four days
\end{tabular}

* The value of 800 fields comes from simulations (the population of which was based on work by Singer et al. 2016), which show that, in $\sim 80$ per cent of the cases with galaxy convolution, the correct field is reached within 800 attempts; for a higher number of fields, the increase in probability per additional field observed becomes minimal.

Table 3. O3 triggers followed-up by Swift. In column 4, (B), (L) and (cWB) note which skymap area is referred to: BAYESTAR, LALInference or coherent WaveBurst respectively. Columns 5 and 6 give the fraction of this area which was covered by XRT observations of non-retracted triggers: 'raw' is the full area, while 'conv.' refers to the area convolved with the relevant galaxy catalogue for the CBC triggers.

\begin{tabular}{lcccccc}
\hline $\begin{array}{l}\text { LVC trigger } \\
\text { ID }\end{array}$ & $\begin{array}{c}\text { Swift obs. } \\
\text { start time (hr) }\end{array}$ & $\begin{array}{c}\text { Obs. } \\
\text { performed }\end{array}$ & $\begin{array}{c}\text { GW 90\% } \\
\text { area/(deg })\end{array}$ & $\begin{array}{c}\text { Frac. of skymap covered } \\
\text { raw }\end{array}$ & $\begin{array}{c}\text { Number of confirmed XRT sources } \\
\text { conv. } \\
\text { Rank 2 }\end{array}$ & $\begin{array}{c}\text { Total } \\
\text { Rank } 3\end{array}$ \\
\hline Rank 4
\end{tabular}

$R$ Trigger was subsequently retracted and no longer thought to be astrophysical.

$\dagger$ The X-ray background for observations of S200115j was strongly elevated due to temperature issues, which caused a large number of spurious sources, many with a high rank. See text for more details. 

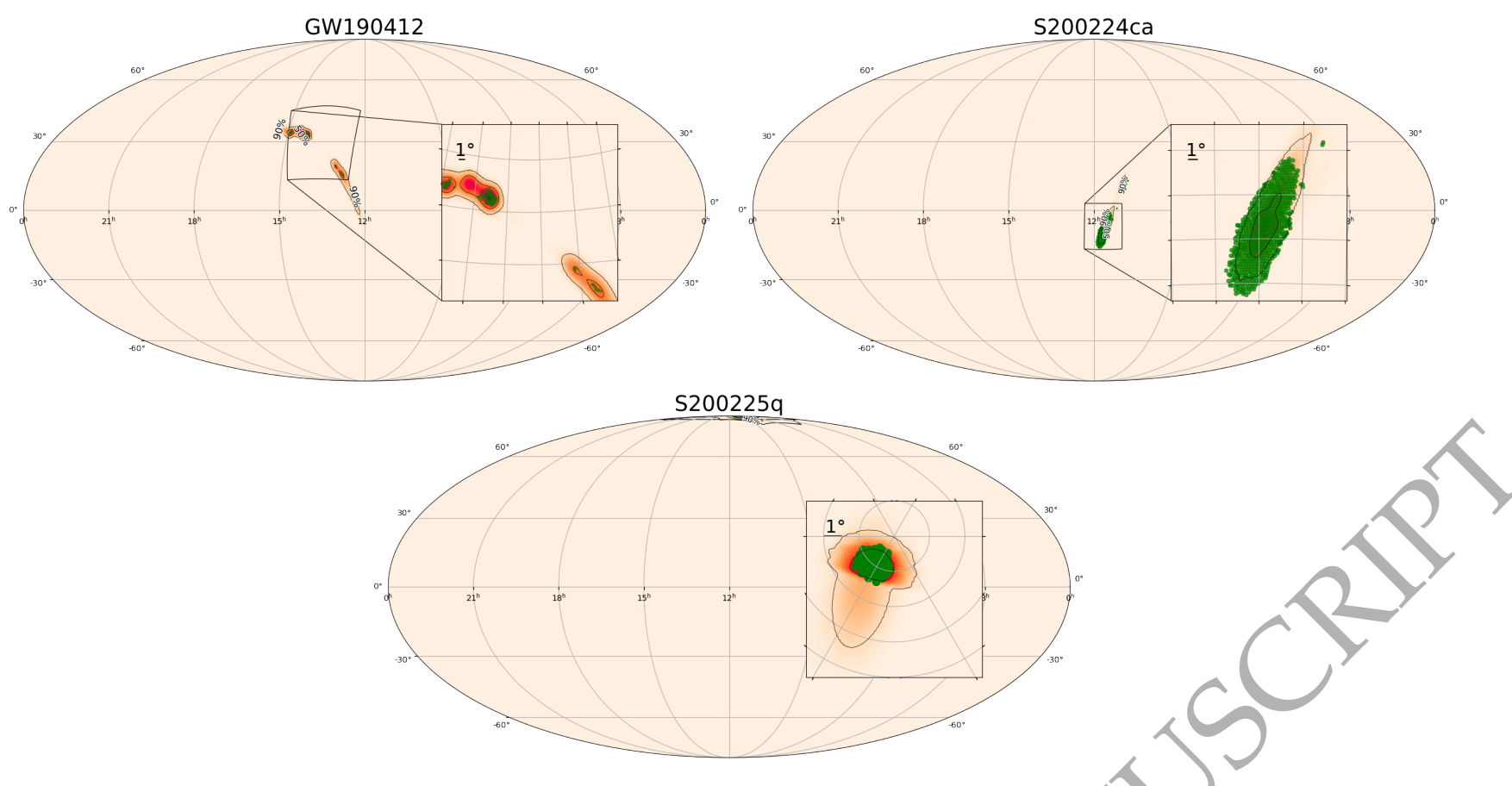

Figure 1. Tiling observations for the BBH triggers. 90 per cent and 50 per cent GW error contours are shown by the solid lines; green spots mark the footprints (location and size) of the XRT fields observed. In the case of S200225q, the fields are all concentrated around the northern pole close to $90 \mathrm{deg}$. in declination, hence a polar view is shown in the inset. S191216ap is not shown, since follow-up of the IceCube error region was performed instead of standard tiling.

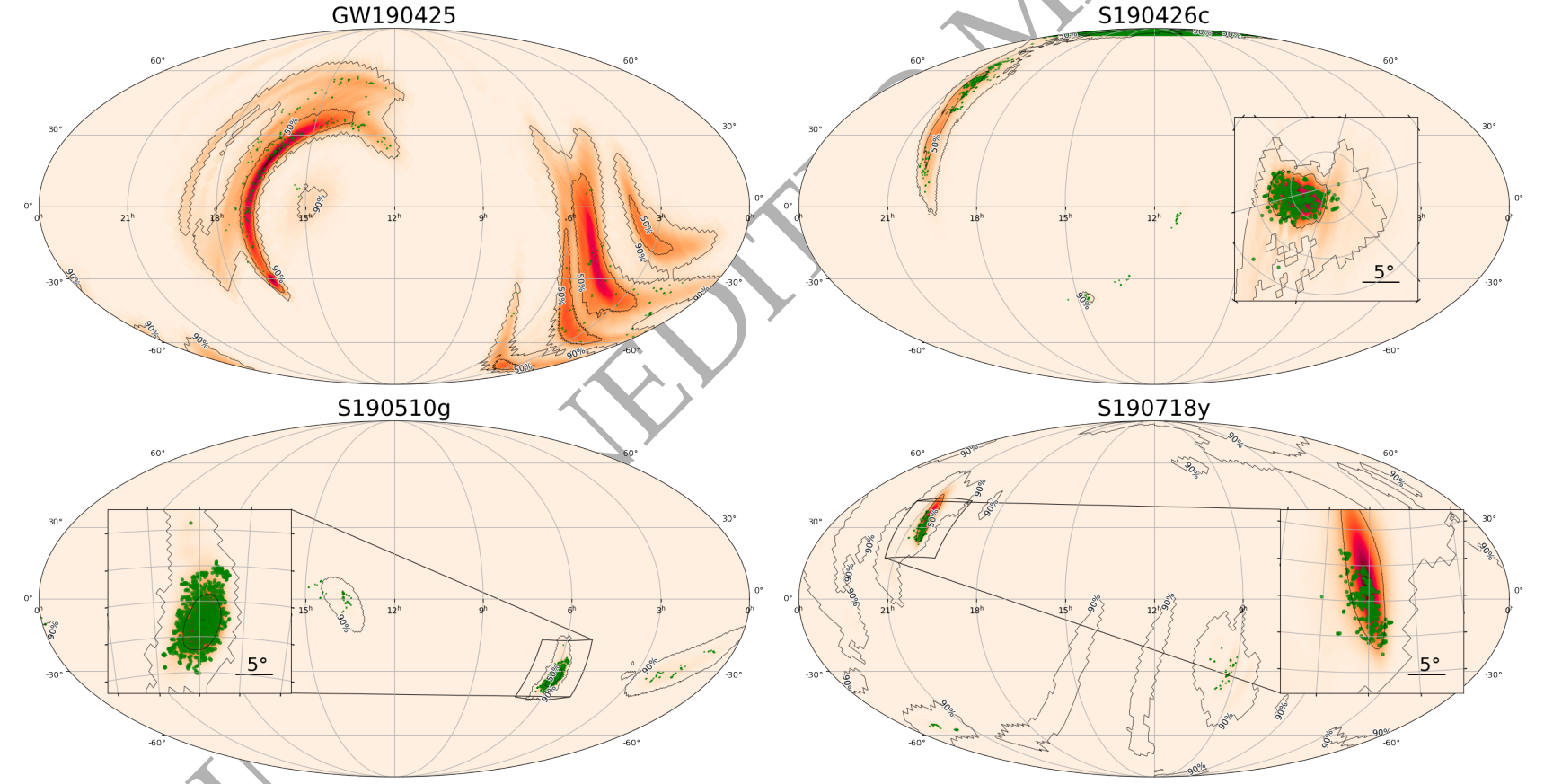

Figure 2. Tiling observations for the BNS triggers. 90 per cent and 50 per cent GW error contours are shown by the solid lines; green spots mark the footprints (location and size) of the XRT fields observed. The inset for S190426c shows a view of the region around 90 deg. declination. Not shown are S191213g and S200213t, for which follow-up of individual externally-discovered sources was performed, rather than a tiling pattern. 

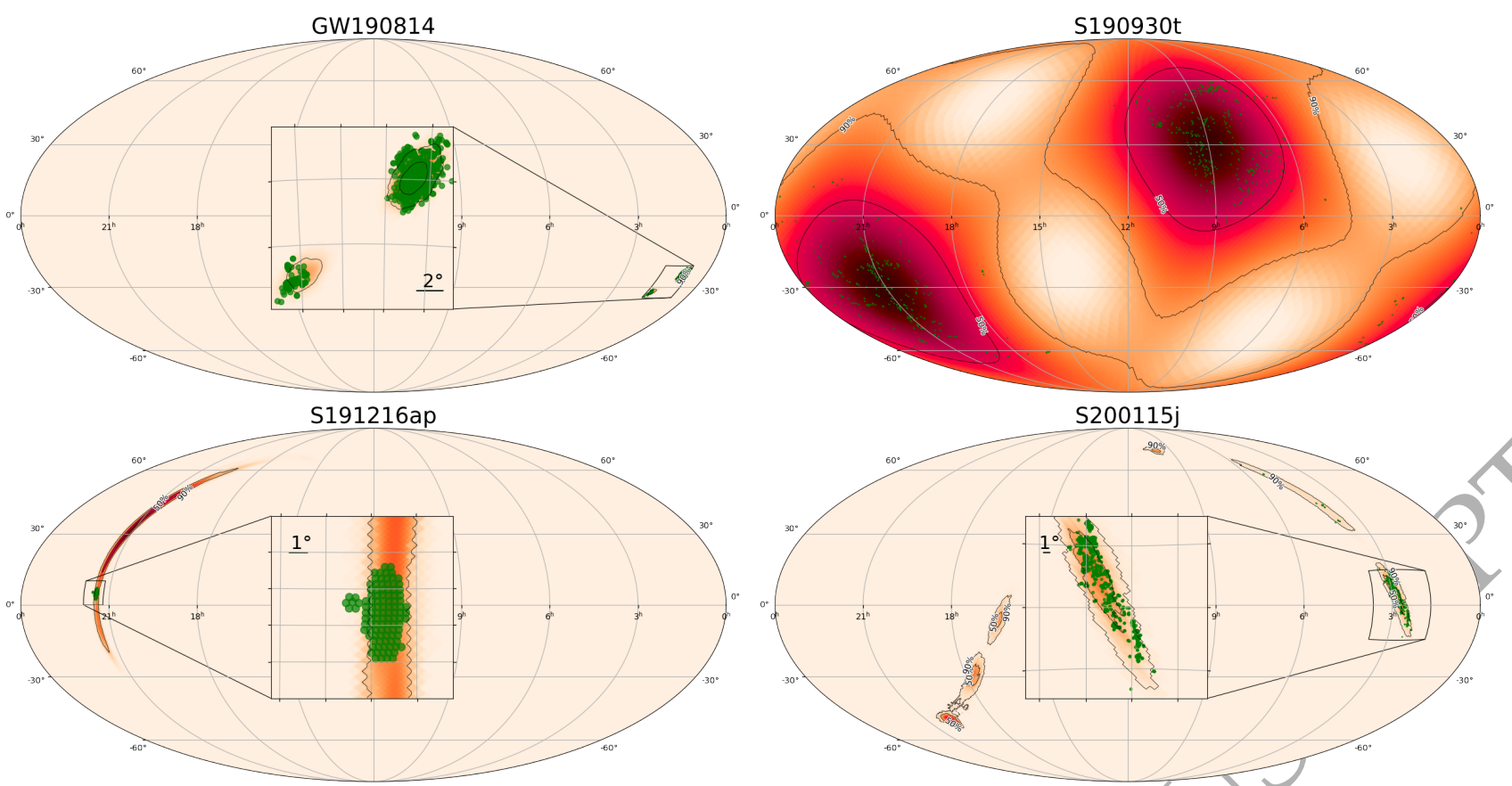

S200115j

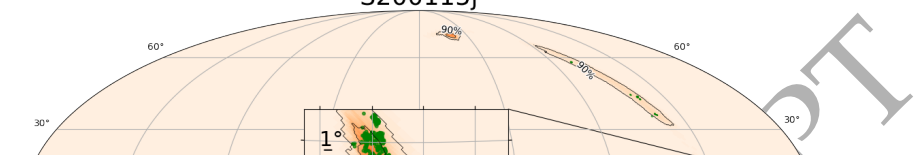

Figure 3. Tiling observations for the NSBH (top row), Mass Gap (middle row) and unmodelled (bottom) triggers. 90 per cent and 50 per cent GW error contours are shown by the solid lines; green spots mark the footprints (location and size) of the XRT fields observed.

\section{RESULTS}

Here we consider each of the O3 GW triggers followed up by Swift, reporting on the actual obseryations performed and the X-ray sources detected in each case. For completeness, even the triggers later retracted have been included. Full details of the fields observed can be found under each specific trigger page at https://www.swift.ac.uk/LVC/, including Swift target IDs, start time of the observation and the exposure for each pointing. Information about each confirmed X-ray source found is also provided there. In addition, the webpages list the X-ray count rate upper limits for UVOT-detected sources with a good Q0 or Q1 quality flag (Oates et al. in prep); given the large number of these sources, we do not list them here, and only refer explicitly to those sources which were deemed worthy of further Swift follow-up. The Gravitational Wave Treasure Map tool $^{12}$ (Wyatt et al. 2020), designed to visualise and coordinate EM follow-up, also includes detailed information about the Swift pointings.

12 http://treasuremap.space/

\section{$3.1 \quad$ S190412m (GW 190412)}

S190412m, a BBH merger at $812 \pm 194$ Mpc, did not satisfy our criteria for follow-up: the area enclosing 90 per cent of the probability was $\sim 151.7 \mathrm{deg}^{2}$, significantly larger than our chosen cut of $10 \mathrm{deg}^{2}$. However, the trigger was used to test a new implementation of the tiling software. 94 pointings of $\sim 80 \mathrm{~s}$ each were performed, covering $9 \mathrm{deg}^{2}$, spanning 38-62.6 ks after the LVC trigger (Fig. 1). Only three X-ray sources were detected, of which two were Rank 3 (uncatalogued X-ray sources with nothing to distinguish them from typical faint background sources; no further follow-up was performed $)^{13}$ and one Rank 4 , correponding to a known Seyfert galaxy.

Following offline analysis, S190412m was confirmed as a highly significant GW detection and renamed GW 190412 (Abbott et al. 2020b).

13 Short follow-up observations of three UVOT candidates were also performed more than a year later in 2020 June; these sources were not detected by XRT, to $\lesssim 0.03$ count $\mathrm{s}^{-1}$. 


\section{$3.2 \quad$ S190425z (GW 190425)}

S190425z was identified as a BNS merger (>99 per cent probability; distance of $156 \pm 41 \mathrm{Mpc}$ ), so was immediately marked for follow-up. The GW candidate was poorly localised since it was essentially a single interferometer trigger (below threshold for the Virgo detector, and the LIGOHanford observatory was offline at the time). 403 observations, covering $46.7 \mathrm{deg}^{2}$ on the sky, were performed between 16-59 ks after the trigger. This covered 6.5 per cent of the LALInference skymap, after convolving with the $2 \mathrm{MPZ}$ galaxy catalogue (Fig. 2). For this GW event, the initial $80 \mathrm{~s}$ tiles were observed, together with target of opportunity (ToO) observations, each of between 1 and $3 \mathrm{ks}$ for four specific sources reported as candidate counterparts in the optical band: ZTF19aarykkb, ZTF19aarzaod (Kasliwal et al. 2019a), AT2019ebq (=PS19qp; Smith et al. 2019) and Swift J170219-122908 (this last source being a possible transient found in an earlier UVOT observation; Breeveld et al. 2019a); these observations occurred 36.5-220.6 ks after the GW trigger. There was also a late time (2020 June) observation of a possible UVOT counterpart (Swift J065827.6454319.8). None of these ToO sources was detected by the XRT, to limits of between $2.5 \times 10^{-3}$ and 0.02 count $\mathrm{s}^{-1}$. ZTF19aarykkb, ZTF19aarzaod and AT2019ebq were subsequently classified as Type II or Ib/IIb supernovae (Perley et al. 2019a; Buckley et al. 2019; Jencson et al. 2019), and therefore unrelated to the GW trigger.

Follow-up 500-s observations were not performed because of S190426c, another GW trigger the following day, taking precedence.

In total, nine X-ray sources were identified: two Rank 3, and seven Rank 4. Of the Rank 4 sources, four are classified as active galactic nuclei (AGN), one a galaxy in a cluster and two were previously listed in ROSAT and XMM-Newton catalogues, at approximately the same flux level as measured here. Of the sources marked as Rank 3, one is identified as a galaxy, and one as an AGN, both classifications based on optical data, with no previous X-ray observations reported.

S190425z is also now officially known as GW 190425 (Abbott et al. 2020a).

\section{$3.3 \quad$ S190426c}

$\mathrm{S} 190426 \mathrm{c}$ had $\mathrm{P}_{\mathrm{BNS}}=0.49$ and $\mathrm{P}_{\mathrm{NSBH}}=0.12$, strongly indicating the merger involved a NS; the distance estimate was $376 \pm 100 \mathrm{Mpc}$. Four months láter, LVC (2019bb) updated the classification of this source to have an increased probability of 0.58 of being terrestrial in origin (c.f. 0.14 when the trigger was first announced). Tiling of 894 unique fields was carried out (both $80-\mathrm{s}$ and 500-s exposures, leading to 1048 pointings in total; Fig. 2), running between $8.6-548 \mathrm{ks}$ after the trigger, detecting $107 \mathrm{X}$-ray sources, of which 68 were Rank 3, and 39 Rank 4 (7 AGN, 18 with no classification other than 'X-ray source' - mainly from ROSAT, 4 galaxies and 10 stars of different types). The observations covered $67 \mathrm{deg}^{2}$ on the sky, equating to 31 per cent of the LALInference skymap after galaxy convolution.

As well as the standard tiling routine, pointed observations of ZTF19aassfws (Perley et al. 2019b) were performed around $1675 \mathrm{ks}$ (20 days) after the trigger, though the source was not detected by XRT (to a $3 \sigma$ upper limit of $1.7 \times 10^{-3}$ count $\mathrm{s}^{-1}$ ); this source has since been retracted as a possible counterpart (Kasliwal et al. 2020b). In addition, XRT source 5 (= 1SXPS J144850.8-400845) in the field was chosen for more follow-up, since it was highlighted as being a factor of $\sim 5.3$ brighter than an earlier, serendipitous observation of that field in 2011 (Evans et al. 2019a). However, the source is ranked as level 4, a known X-ray source consistent with being an AGN. This slight brightening is therefore most likely to be an AGN flare (see Section 4 for further discussion of AGN activity). Swift J201946.1+594818, a source detected in the initial UVOT tiling, was also re-observed as a potential counterpart, though subsequently decided not to be of interest (Kuin et al. 2019c); it was not detected by XRT down to $3.9 \times 10^{-3}$ count s$^{-1}$.

\section{$3.4 \quad \mathrm{~S} 190510 \mathrm{~g}$}

S190510g initially had a high (0.97) chance of being a BNS system, at a distance of $227 \pm 92 \mathrm{Mpc}$. However, the classification was updated the following day to $\mathrm{P}_{\mathrm{BNs}}=0.42$ and $\mathrm{P}_{\text {Terres. }}=0.58$. Swift observed 977 fields (mainly $\sim 80 \mathrm{~s}$ tiles) between $7.2-270 \mathrm{ks}$, covering $76.9 \mathrm{deg}^{2}$ on the sky (corresponding to 67 per cent of the probability in the galaxy-convolved LALInference skymap; Fig. 2). Follow-up was aborted once the classification of a BNS merger became less likely. $33 \mathrm{X}$-ray sources were detected, with all but five being previously known (the remaining five were Rank 3). Of the 28 Rank 4 sources, nine are classified as AGN, 11 as galaxies (or a cluster of galaxies), six are different types of stars, and the remaining two are unknown X-ray sources in ROSAT catalogues.

\subsection{S190718y}

This trigger had a 0.97 probability of being a terrestrial noise event; however, were it to be real, then the probability was that it was formed through a binary NS merger; following the initial decision tree, any GW events which were flagged as containing a NS would be followed up. Therefore, 368 pointings were performed (both 80 -s, where some were repeated, and 500-s exposures) from $13-365 \mathrm{ks}$ after the trigger, covering $30.9 \mathrm{deg}^{2}$ (22 per cent of the probability in the BAYESTAR skymap after convolution; Fig. 2). If this event were cosmological, its distance is estimated to be $226 \pm 164$ Mpc. A total of 45 X-ray sources were found, with 27 Rank 3 and 18 Rank 4 . Of these known sources, three are AGN, five galaxies, five are unidentified ROSAT X-ray sources, four are stars, and one has no associated classification.

\section{$3.6 \quad$ S190728q}

Because of the large positional uncertainty from the LVC, the BBH S190728q (at a distance of $785 \pm 212 \mathrm{Mpc}$ ) did not pass the Swift filtering criteria for follow-up. However, IceCube (Aartsen et al. 2017) announced a neutrino candidate (IceCube Collaboration 2019a,b,c); with this better (though still large: radius of $4.8 \mathrm{deg}$ ) possible localisation, follow-up observations were approved (exposure times of $\sim 100 \mathrm{~s}$ per tile), and ran for $45-104 \mathrm{ks}$ after the LVC trigger, covering $14.3 \mathrm{deg}^{2}$ on the sky (only 0.6 per cent of the galaxy-convolved BAYESTAR 
skymap, though $\sim 20$ per cent of the IceCube localisation; Tohuvavohu et al. 2019c). Within these observations, three Rank 4 sources were identified, corresponding to an active galaxy, a star and an infrared (IR) source.

A ToO observation of ZTF19abjethn/AT2019lvs (Kasliwal et al. 2019b) was also performed, finding a corresponding X-ray source at a level of $2.9_{-1.1}^{+1.4} \times 10^{-3}$ count s $^{-1}$. This source was subsequently noted to be outside the updated skymap, and thus retracted as a potential counterpart (Kasliwal et al. 2019c); Smartt et al. (2019) and Magee et al. (2019) also classified it as a cataclysmic variable.

\subsection{S190808ae - Retracted}

S190808ae was initially announced as a CBC trigger with $\mathrm{P}_{\mathrm{BNS}}=0.42$ and $\mathrm{P}_{\text {Terres. }}=0.57$. A retraction was issued by the LVC around $6 \mathrm{hr}$ later (LVC 2019at), by which time a series of 80-s observations had already begun with Swift. In total, 36 pointings were performed between $12-19 \mathrm{ks}$, detecting two known galaxies.

\subsection{S190814bv (GW 190814)}

While S190814bv was initially classified as a Mass Gap trigger with a large area (370 $\mathrm{deg}^{2}$ of the convolved skymap enclosing the 90 per cent probability, $\mathrm{P}_{0.9}$ ), an updated BAYESTAR skymap about $1.5 \mathrm{hr}$ later decreased this error substantially such that $\sim 18 \mathrm{deg}^{2}$ would enclose $\mathrm{P}_{0.9}$. While this still did not satisfy the standard Swift follow-up criteria (Table 1), a judgement call was made to observe anyway. In addition, the classification was updated the following day to NSBH, together with a further refinement of the error region with a LALInference skymap (and distance of $267 \pm 51 \mathrm{Mpc}$; LVC 2019av), further supporting. our planned follow-up, given that the system likely contained a NS. Significant ground-based follow-up was performed of this trigger (e.g., Ackley et al. 2020; Thakur et al. 2020; Andreoni et al. 2020b).

Swift observed 352 fields from 11-471 ks (both initial 80 -s tiles, many of which were repeated, and later $500-\mathrm{s}$ pointings; in total, 529 observations were taken); these covered $20.3 \mathrm{deg}^{2}$, corresponding to 89 per cent of the galaxyconvolved LALInference skymap (Fig. 3), or 78 per cent of the earlier BAYESTAR map. In addition to the standard tiling algorithm, observations were planned to cover rising radio source and optical transient AT 2019osy (also known as ASKAP 005547-270433) which had been announced (Stewart et al. 2019; Andreoni et al. 2019a; Dobie et al. 2019); this source was not detected in X-rays (upper limit of $2.0 \times 10^{-3}$ count $\mathrm{s}^{-1}$; also undetected by Chandra: Jaodand et al 2019), with only the nearby galaxy visible in the UVOT data (Evans et al. 2019e). In total, 94 X-ray sources were detected, with 32 Rank 4, 60 Rank 3 - and two Rank 2 sources (that is, sources marked as possible afterglows), sources 2 and 99 in the field.

Upon further investigation, sources 2 and 99 were found to be the same source, not correctly aggregated due to a bug in the automated analysis software. They were highlighted as potentially interesting due to being almost $9 \sigma$ (a flux ratio of 2.9 ) brighter than the catalogued count rate in the 1SXPS catalogue (1SXPS J005446.7-245528 = 2SXPS J005446.7245530). Follow-up observations initially suggested a possible fading trend, though the latest data, collected 13.5 days after the trigger, showed the source to have rebrightened again. While not a previously catalogued X-ray source, the position is consistent with a known AGN which could easily explain the variability. This source is clearly detected in the UVOT data, with an AB magnitude of $u \sim 17.1-17.2$ during the observations.

A number of the Rank 3 and 4 sources in the field were also considered of possible interest, due to their potential variability, and were therefore re-observed by Swift. None of the follow-up observations of the Rank 3 sources (sources 14, $31,51,59,74$ and 88) identified significant fading, so they were dropped from further consideration.

Source 6 matches XMMSL2 J005323.1-244018 in the $X M M-N e w t o n$ slew catalogue, though is significantly fainter $\left(\sim 0.1\right.$ count $\mathrm{s}^{-1}$ compared with the catalogued rate of $\sim 0.8$ count $\mathrm{s}^{-1}-$ both in terms of XRT counts). There was a slight indication that the source was rising, so additional observations were taken. However, the count rates remained consistent within the error bars.

Source 7, a previously catalogued ROSAT source called 1RXS J005355.4-240439, was 2.6 $\sigma$ (flux ratio of 5.2) brighter than the catalogued level, and also fading at $3.2 \sigma$. This fading is noted between a single point and all the later measurements which are consistent with the ROSAT measurement. This source is also catalogued as a possible AGN.

Source 43 , also detected as a bright source by ROSAT (1RXS J005040.5-254115), faded at 2.0 $\sigma$ between one higher measurement (consistent with the catalogued rate) and the subsequent fainter detections. This source is a known AGN.

Of the 32 known Rank 4 sources (including those discussed explicitly above), ten are classified as AGN, seven each as galaxies and ROSAT X-ray sources of unspecified type, two are stars, one is an ultra-luminous $\mathrm{X}$-ray source, one a supernova remnant, with the final four catalogued as unknown types of X-ray sources detected by other missions.

Swift observations of S190814bv will be described in more detail by Cenko et al. (in prep.). Following confirmation of its reality as a merger of a $\mathrm{BH}$ with some form of compact object (either the lightest black hole or the heaviest NS yet discovered), at $241_{-45}^{+41} \mathrm{Mpc}$, this trigger is also known as GW 190814 (Abbott et al. 2020c).

\section{$3.9 \quad$ S190822c - Retracted}

After an initial classification as a BNS, prompting Swift observations, S190822c was retracted as an astrophysical source about $45 \mathrm{~min}$ later (LVC 2019ax). However, before this updated information, a 37 point tiling observation $(80 \mathrm{~s}$ per tile) had been uploaded to the spacecraft, and ran from 7.3-15 ks after the trigger. An AGN and a previously unknown X-ray source were found in these data.

\subsection{S190930t}

S190930t was marked as 74 per cent likely to be a NSBH merger at a distance of $108 \pm 37 \mathrm{Mpc}$; the LVC error region was very large, covering the majority of the sky, since it was a single interferometer detection. Swift observed 735 
different fields between $7.6-120 \mathrm{ks}$, covering $83.1 \mathrm{deg}^{2}$ of the sky, and 2.9 per cent of the convolved BAYESTAR skymap (Fig. 3). After the initial 80-s tiles, further observations were performed of a number of new sources identified in the early XRT (one source of interest) and UVOT (ten sources) data, as well as the externally-detected AT2019rpn (also known as ZTF19acbpqlh; Stein et al. 2019a), which was not detected in XRT observations, down to an upper limit of $5.3 \times 10^{-3}$ count $\mathrm{s}^{-1}$; this source was later classified as a type II supernova, unrelated to S190930t (Kasliwal et al. 2020b). A final UVOT candidate was observed in 2020 June, but was undetected in XRT data $\left(<0.025\right.$ count $\left.\mathrm{s}^{-1}\right)$.

From the XRT perspective, source 12 was initially considered as potentially interesting because it was detected at about twice the RASS upper limit (though with a substantial error bar) and faded slightly (at the $1.4 \sigma$ level) between the initial observation at $\sim 34$ ks after the GW trigger and later observations starting at $\sim 240 \mathrm{ks}$ (Evans et al. 2019f). Beyond this time, the count rate remained around 0.01 count $\mathrm{s}^{-1}$. Again, despite not having a previous X-ray detection, the position is coincident with a known AGN.

Of the ten UVOT sources flagged to be followed up, one was thought to correspond to source 28 in the XRT list; this only produced an unconstraining upper limit in the initial $80 \mathrm{~s}$ snapshot, but was then detected in longer follow-up observations, with a count rate varying between 0.009 and 0.03 count $\mathrm{s}^{-1}$. This source, seen to be fading in the UVOT, was subsequently named AT2019sbk (Tohuvavohu et al. 2019d). With additional data, the localisation of the X-ray source was improved, and determined to be unrelated to AT2019sbk, but instead consistent with the centre of the galaxy 2MASX J22471856-5814422 (Oates et al. 2019e).

None of the other potentially interesting UVOT sources was detected by XRT, to a typical upper limit of $\sim 4-$ $5 \times 10^{-3}$ count s$^{-1}$. At the time of writing (around 300 days post-trigger), Swift J221951-484240 (a candidate transient identified in the UVOT data; Oates et al. 2019c,d) is still being regularly observed (Oates et al. in prep); given the larger amount of data, the X-ray upper limit for this source is deeper: $2.6 \times 10^{-4}$ count $\mathrm{s}^{-1}$.

From all the tiling observations of this trigger, five Rank 3 and 11 Rank 4 sources were detected, of which five are known AGN, three are stars, one is a galaxy and the remaining two are previously catalogued ROSAT X-ray sources, showing no sign of outburst.

\subsection{S191110af - Retracted}

The first O3b trigger Swift observed was retracted four days after the event (LVG 2019bz). Initially classified as an unmodelled trigger with a central frequency of $\sim 1.8 \mathrm{kHz}$, this was flagged as a possible Galactic event, so 80-s Swift observations were planned. After convolving the error region with the Galactic plane given the trigger type, 798 fields were observed, from 10-203 ks after the trigger, finding six Rank 3 and 11 Rank 4 sources. Unsurprisingly given the concentration around the Galactic plane, five of these sources were marked as stars, and two as high-mass X-ray binary systems. There were also two catalogued, though unidentified, $\mathrm{X}$-ray sources and two known to be IR emitters.

\section{$3.12 \quad$ S191213g}

Although S191213g was marked as likely to be a BNS merger (76 per cent; distance of $200 \pm 80 \mathrm{Mpc}$ ), the large error region meant that tiling by Swift would only cover 0.017 of the area in 24 hours, well below the chosen limit of 0.1. While no tiling was therefore performed, observations of three ZTF sources (ZTF19acykzsk, ZTF19acyldun, ZTF19acymixu; Andreoni et al. 2019b; Stein et al. 2019b) and a Pan-STARRS candidate (PS19hgw/AT2019wxt; McBrien et al. 2019) did take place over the following few days (Oates et al. 2019f,g). None of these sources was detected by the XRT, with limits of $\sim 4.5-6.5 \times 10^{-3}$ count $\mathrm{s}^{-1}$, and were all later classified as supernovae unrelated to the GW event (Kasliwal et al. 2020b; Valeev et al. 2019)

\section{$3.13 \quad$ S191216ap}

S191216ap was a Mass Gap trigger, with a low (though nonzero) probability of hosting a disrupted NS, at $375 \pm 70$ Mpc. The error region was large, meaning Swift tiling would only have covered $\sim 0.33$ of the area in $24 \mathrm{hr}$, whereas the follow-up criteria require $\mathrm{P}_{24 \mathrm{hr}} \gtrsim 0.5$. However, IceCube announced a counterpart neutrino candidate (IceCube Collaboration 2019d,e), and Swift performed 100 tiles (of $\sim 50-60 \mathrm{~s}$ each) to cover the convolution of the neutrino and GW error regions, spanning $22-42 \mathrm{ks}$ after the trigger and covering $10.2 \mathrm{deg}^{2}$ on the sky (5.8 per cent of the BAYESTAR skymap after convolution with the galaxy catalogue, and 65 per cent of the probability contained within the combined GW and neutrino localisations; Fig. 3; Evans et al. 2019h). In addition to this, HAWC (HighAltitude Water Cherenkov observatory; Springer et al. 2016) detected a sub-threshold event with a position similar to that of the IceCube one, though not covered by the initial Swift tiling (HAWC Collaboration 2019). Therefore, a further 7-point tiling pattern (500 s per tile; this fully covered their 68 per cent containment region) was observed, as well as specific pointings towards the nine galaxies mentioned by Singer et al. (2019) as being coincident with the LIGO/Virgo and HAWC positions (Evans et al. 2019i). In total, 20 XRT sources were found: 14 Rank 3 and 6 Rank 4. Of the six previously-known sources, one is an AGN, three are galaxies and the remaining two are associated with known radio and IR/UV sources.

\subsection{S200114f}

S200114f was a low-frequency $(\sim 65 \mathrm{~Hz})$ unmodelled trigger. Given that the error region was relatively small, 80-s Swift observations were planned, with 206 tiles spanning 6.6-99 ks after the trigger; the trigger S200115j on the following day then took precedence. This covered $21.9 \mathrm{deg}^{2}$ on the sky, corresponding to 30 per cent of the probability in the cWB skymap (Fig. 3). The BAT FOV covered almost 98 per cent of the LVC probability (Palmer et al. 2020a) for this trigger; no counterpart candidates were identified.

Eight X-ray sources were found, including one flagged as a strong candidate to be the EM counterpart to the GW trigger (initially a Rank 2 source, later promoted to Rank 1 as the error bars on the integrated flux improved with 
more data); the others consisted of six Rank 3 and a single Rank 4 source (a rotationally-variable star also detected by ROSAT).

The source of interest ('source 2') was so flagged because it showed early indications of fading (Evans et al. 2020a,b). However, this source is spatially coincident with a known AGN. Repeated follow-up observations of the source were taken by Swift, to investigate its evolution. After the initial brief fading from 0.1 to 0.02 count s $^{-1}$ around $52-53 \mathrm{ks}$ after the trigger, the source stayed consistently $\sim 0.06$ count $\mathrm{s}^{-1}$; this was still the case when the source became too close to the Sun for Swift to observe, more than four months after the trigger. The corresponding UVOT source also showed no signs of variability, with $u \sim 16.9$.

We note that the new Gamma-ray Urgent Archiver for Novel Opportunities (GUANO; Tohuvavohu et al. 2020) system for Swift-BAT was activated by the S200114f event, leading to good limits of $<8.1 \times 10^{-8} \mathrm{erg} \mathrm{cm}^{-2} \mathrm{~s}^{-1}$ ( $8 \sigma$ confidence level; $14-195 \mathrm{keV}$ ) being placed on a BAT prompt gamma-ray detection within \pm 15 s of the GW trigger.

Swift follow-up of S200114f, including source 2, will be analysed in more detail by Evans et al. (in prep.).

\subsection{S200115j}

S200115j was classified as a Mass Gap event, with a high probability of containing a disrupted NS, at a distance of $340 \pm 79$ Mpc. Swift observations covered 512 unique fields spanning 7.1-501 ks after the trigger: both the initial phase of 80-s observations (some of them repeated), and the longer 500 -s exposures (for most of the fields) were performed, leading to 719 pointings in total. The localisation skymap changed considerably between the initial BAYESTAR and later LALInference maps, with the error region shifting and decreasing in size; the Swift observations were planned and initiated when only the BAYESTAR maps were available. In total, $36.2 \mathrm{deg}^{2}$ of the sky were covered, corresponding to 9.7 per cent of the galaxy-convolved updated LALInference skymap. Fig. 3 shows the tiles plotted over the LALInference map.

During the Swift observations, the XRT experienced an extended interval of higher than normal operating temperature. This led to increased instrumental background, and the issuing of automatic GCN notices for spuriously highranked sources (Ranks 1 and 2; Evans 2020). As always, each source was vetted by a human, and any obviously spurious sources not promoted to the public page.

In total, XRT detected 82 sources we believe are likely to be real. Of these, nine are Rank 2 (sources of interest), 41 Rank 3, and 32 Rank 4. Looking into the apparently interesting sources in more detail/see also Oates et al. 2020a; Evans et al. 2020d), it was found that sources 130, 488, 717 and 748 all correspond to (likely) AGN, while source 136 matches a 2MASS galaxy and 745 an emission line galaxy (Mrk 1036); additional observations over the following days and weeks showed nothing to distinguish them from ordinary AGN activity in these sources. There were corresponding UVOT $u$-band detections of each of these except source 488 , but no significant evidence for variability in any of them.

Source 487 was noted as being above the RASS limit and fading, but this description is based on a single detection during the interval of high background (all other ob- servations provided upper limits only), so is likely spurious; there was no detection by UVOT to a $3 \sigma$ limit of $u>20-21$. Source 707 was above the RASS detection limit, and faded between two detections (all other observations, out to 175 days after the trigger, were upper limits, suggesting possible further fading). Given that the source is relatively faint, even an extra photon or two from the high background could be skewing these results. Additionally, the source was originally only flagged as 'reasonable' (see Evans et al. 2014, for a definition of the detection flags), meaning that there is a $\sim 7$ per cent probability of the source being spurious. There was no UVOT source detected at this location $(u>21-22)$, although these observations did not start until 11 days after the GW trigger. In a similar vein, source 746 showed fading from above to below the RASS limit between two observations (although the second data point only contained three source counts; the background level is low enough that, using Bayesian statistics, this is still a strongly significant detection of $>99.999$ per cent), with additional upper limits in between, and later limits (out to $\sim 180$ days post-trigger) implying the source had faded further. No counterpart was detected by UVOT to $u>20$.

Of the 32 Rank 4 known, sources, 18 are AGN, 4 are galaxies, 3 are X-ray sources of an unknown type, 3 correspond to stars (including one RS CVn type) and the remaining four are simply catalogued as IR, UV or 'blue' sources.

\section{$3.16 \quad$ S200213t}

S200213t was a BNS trigger (distance of $200 \pm 80 \mathrm{Mpc}$ ), but with too large an area to satisfy Swift follow-up criteria. However, following the announcement of a neutrino candidate from IceCube (IceCube Collaboration 2020), a sevèn-point tiling plan was uploaded $(\sim 1.3 \mathrm{ks}$ per tile; Countryman et al. 2020). Additionally, ToO observations of ZTF20aanakcd (also known as AT2020cmr; Reusch et al. 2020) and ZTF20aamvmzj (also known as AT2020cja; Kasliwal et al. 2020a), possible optical counterparts to the GW event, were performed (Oates et al. 2020b,c). In total nine separate fields were observed, running from 20 $22.5 \mathrm{ks}$ after the trigger (for the seven point tiling; observations of ZTF20aamvmzj/AT2020cja continued until a month after the trigger), and covering $1.2 \mathrm{deg}^{2}$ on the sky (0.01 per cent of the galaxy-convolved LALInference map). While neither of the ToO sources was detected by the XRT $\left(<3.1 \times 10^{-3}\right.$ and $6.7 \times 10^{-4}$ count $\mathrm{s}^{-1}$ for ZTF20aanakcd and ZTF20aamvmzj/AT2020cja, respectively), five Rank 3 field sources were identified. ZTF20aanakcd was later found to be a type IIn supernova and unrelated to S200213t (Andreoni et al. 2020a).

\subsection{S200224ca}

S200224ca was announced as a BBH at a distance of $1574 \pm 322 \mathrm{Mpc}$. It was well localised, such that 90 per cent of the convolved probability covered only $72 \mathrm{deg}^{2}$ of the sky (Fig. 1), meaning that $\mathrm{P}_{24 \mathrm{hr}}=0.66$ for Swift, within our monitoring criteria. Swift observed 670 separate fields, from 21-196 ks after the trigger, covering $64.5 \mathrm{deg}^{2}$ on the sky, corresponding to 79 per cent of the probability in the convolved LALInference skymap. Most of the observations were 
the initial 80-s tiles; as the second phase of 500-s tiles was begun, a new GW trigger, S200225q, was announced, and took precedence. Six UVOT sources were also followed-up with further ToO observations, but all were undetected in $\mathrm{X}$-rays (Oates et al. in prep).

Within the tiling observations, only eight X-ray sources were identified, two Rank 3 and six Rank 4 (all of which are consistent with catalogued AGN).

In 2020 May, the unknown X-ray sources 5 and 9 were further observed. Source 5 showed no real evidence for fading, whereas source 9 had faded between the observations performed 1.6 and 90-120 days after the GW trigger (the later observations providing upper limits; there were only four source counts in the initial detection, however, and the source is only flagged as 'reasonable').

This trigger will be individually discussed in a future publication (Klingler et al. in prep.).

\section{$3.18 \quad$ S200225q}

S200225q, at a distance of $994 \pm 187 \mathrm{Mpc}$, was strongly (95 per cent) associated with a BBH merger, although the FAR of $1 / 3.5$ yr was higher than the cut-off chosen for standard Swift follow-up. However, given the very good updated localisation (50 per cent area of only $3 \mathrm{deg}^{2}$, leading to $\mathrm{P}_{24 \mathrm{hr}}=0.87$ of the convolved map) released $38 \mathrm{hr}$ later (LVC 2020x), a decision was made to follow-up. The initial plan of performing a 37 point tiling was interrupted by a trigger on GRB 200227A (Laha et al. 2020). Restarting the tiling later, a total of 70 observations of $\sim 80 \mathrm{~s}$ each were performed, from $172-224 \mathrm{ks}$ after the trigger, and covering $3.8 \mathrm{deg}^{2}$ on the sky. This covered 51 per cent of the galaxy-convolved LALInference skymap (Fig. 1). Because the follow-up was interrupted and delayed, the second phase of $500 \mathrm{~s}$ tiles was not performed. Only a single X-ray source was detected (Evans et al. 2020f), corresponding to a previously-catalogued ROSAT object.

\section{DISCUSSION}

During the third LIGO/Virgo observing run, Swift followed up 18 of the GW triggers announced, three of which were subsequently retracted, performing almost 6500 separate pointings. Of the 15 non-retracted triggers, four were classified as likely BBHs, six as BNS mergers, two NSBHs, two Mass Gap events and one an unmodelled (Burst) trigger. In total, four O3 triggers (GW 190412, 190425, 190521 and 190814) have been officially confirmed as being real GW events. While Swift détected many X-ray sources during these observations, none stands out as a likely EM counterpart to a GW event - that is, a new bright (or significantly brightened) source, close to a known galaxy (see description of source rankings in Section 2).

\subsection{Sources}

Much of the cosmic X-ray background can now be resolved as emission from discrete AGN (e.g. Shanks et al. 1991; Barcons et al. 2007; Caccianiga et al. 2008; Mateos et al. 2008; Corral et al. 2014; Oh et al. 2018), and such active galaxies are inherently variable at X-ray (and other) wavelengths, over timescales from as short as minutes up to many years (e.g., McHardy 1985; Barr \& Mushotzky 1986; Mushotzky et al. 1993; Boller, Brandt \& Fink 1996; Giommi et al. 2019). It is therefore unsurprising that widefield observations such as those performed in the follow-up of large GW error regions reveal variable X-ray sources, under the assumption that many of these unknown sources are AGN. Considering the 18 LVC (15 likely real, three retracted) triggers followed-up by Swift during O3, 198 catalogued (Rank 4) sources were found. Of these, a third (66) are known AGN. In addition, of the 11 unique sources which were flagged as potentially interesting (i.e., Rank 1 or 2), eight correspond to AGN (the remaining three were uncatalogued). A total of 243 previously uncatalogued Rank 3 sources were detected, and it is very likely that many of these will be AGN. Of the remaining Rank 4 sources, the next largest population corresponds to galaxies (some in clusters), with 34 of the sources being classified as such.

Graham et al. (2020) report a possible EM counterpart for the (probable) BBH merger S190521g (not followed-up by Swift because of the large area), which is consistent with the merger occurring in, and interacting with, the accretion disc of an AGN. This suggests that perhaps more attention should be paid to following up AGN in the GW error regions in future runs; knowledge of whether emission from a given active galaxy typically remains close to constant, before showing an unusualflaring event close in time to a GW trigger (as was the case for J124942.3+344929/ZTF19abanrhr in Graham et al 2020), would be useful, though may be difficult to achieve.

\subsection{Flux distribution}

Fig. 4) shows histograms of the peak fluxes of the uncatalogued and catalogued sources detected by the XRT during the $\mathrm{O} 3$ observing run. These $0.3-10 \mathrm{keV}$ absorbed fluxes are estimated from the measured peak count rate by assuming a power-law spectrum with photon index $\Gamma=1.7$ and an absorbing column of $\mathrm{N}_{\mathrm{H}}=3 \times 10^{20} \mathrm{~cm}^{-2}$ (a conversion factor of $\sim 4.3 \times 10^{-11} \mathrm{erg} \mathrm{cm}^{-2}$ count $^{-1}$ ). Note that the fluxes plotted are the peak values from the observations.

Unsurprisingly, the uncatalogued sources are skewed towards lower fluxes than the catalogued ones, with the majority of the sources detected having an observed $0.3-10 \mathrm{keV}$ flux of around $5 \times 10^{-13} \mathrm{erg} \mathrm{cm}^{-2} \mathrm{~s}^{-1}$; the previously catalogued source number peaks about an order of magnitude brighter than this. The median values are more similar, at $\sim 9.9 \times 10^{-13}$ and $1.7 \times 10^{-12}$ for uncatalogued and catalogued sources, respectively. The uncatalogued sources range in flux from $1.0 \times 10^{-13}$ to $6.6 \times 10^{-12} \mathrm{erg} \mathrm{cm}^{-2} \mathrm{~s}^{-1}$, while the known sources cover $1.6 \times 10^{-13}$ to $9.6 \times 10^{-11}$ erg $\mathrm{cm}^{-2} \mathrm{~s}^{-1}$, factors of $\sim 60$ and 600 between brightest and faintest, respectively. Only one of the catalogued sources is brighter than $5 \times 10^{-11} \mathrm{erg} \mathrm{cm}^{-2} \mathrm{~s}^{-1}$, though: source 1 in the field of S190510g, which is known to be a quasar (QSO B0548-322). Excluding this source, the ratio of brightest to faintest catalogued sources is $\sim 200$.

In comparison, the 2SXPS catalogue has a median 0.3 $10 \mathrm{keV}$ flux of $4.7 \times 10^{-14} \mathrm{erg} \mathrm{cm}^{-2} \mathrm{~s}^{-1}$, more than a factor of ten lower; however, the mean exposure time for an observation in 2 SXPS is $\sim 2 \mathrm{ks}$, much longer than the $\sim 80$ 


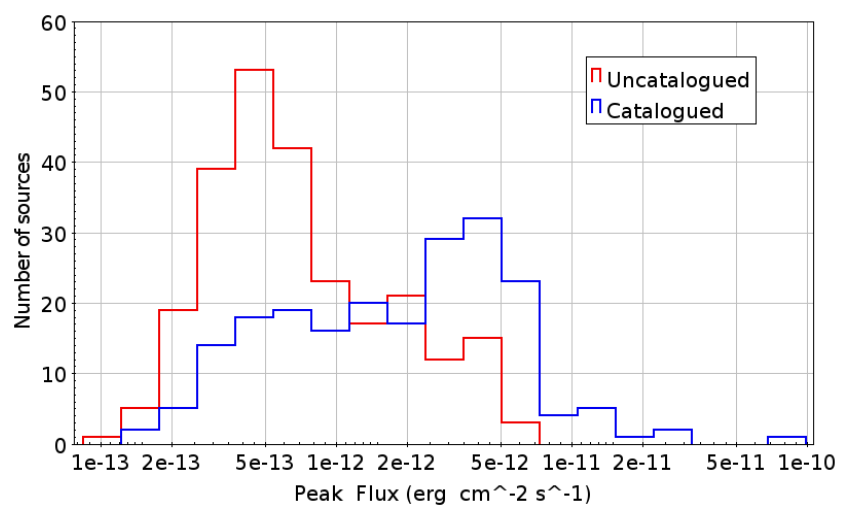

Figure 4. Histogram of the peak X-ray fluxes $(0.3-10 \mathrm{keV})$ for the uncatalogued and catalogued X-ray sources across all GW follow-up during O3.

$500 \mathrm{~s}$ exposures obtained during the GW tiling (Evans et al. 2020g).

\subsection{Detection limits}

No strong candidates for X-ray counterparts to any of the O3 GW triggers were identified. While different triggers were observed for different amounts of time (though the majority of fields were observed for $\sim 80 \mathrm{~s}$ ), it may still be instructive to provide typical upper limits on source detections. For each (non-retracted) GW event where the standard largescale tiling was performed, we estimate the mean $3 \sigma$ upper limit on the X-ray count rate by averaging the XRT nondetections for the UVOT Q0/Q1 sources for that trigger; since the UVOT sources are scattered throughout the area covered, this should provide a good estimate of the limiting brightness for X-ray sources in each GW error region. These values are given in Table 4; the same conversion factor as above from count to flux units was used. The average 0.3 $10 \mathrm{keV}$ flux upper limit across all the fields is $3.60 \times 10^{-12}$ erg $\mathrm{cm}^{-2} \mathrm{~s}^{-1}$. The $\mathrm{O} 3 \mathrm{GW}$ triggers (BBH, NSBH and BNS combined) span a large range of estimated distances, from 108 to $1574 \mathrm{Mpc}$ (S190930t and S200224ca, respectively), with a mean value of $474 \mathrm{Mpc}$. For BNS events - for which a short GRB-like counterpart is most likely - the GW network sensitivity was $\sim 140 \mathrm{Mpc}^{14}$, hence we scaled GRB light curves to this distance in Fig 5. The corresponding average luminosity upper limit is therefore $\sim 10^{44} \mathrm{erg} \mathrm{s}^{-1}$ (for a distance of $474 \mathrm{Mpc}$ ), or $\sim 10^{43} \mathrm{erg} \mathrm{s}^{-1}$ (at $140 \mathrm{Mpc}$ ).

Figure 5 shows the median flux light-curve for short GRBs based on the flux-limited sample of D'Avanzo et al. (2014), shifted to a distance of $140 \mathrm{Mpc}$, and plotted against days since trigger. For GRBs without a measured redshift, the average value of $z=0.85$ was assumed. This plot shows that, for a typical on-axis short GRB, we would expect the X-ray afterglow to be above $3.60 \times 10^{-12} \mathrm{erg} \mathrm{cm}^{-2} \mathrm{~s}^{-1}$ for around $3_{-2.5}^{+50} \mathrm{~d}$ after the trigger, and therefore readily detectable by XRT observations.

14 See https://www.gw-openscience.org/detector_status/ day/20200227/, where the last part of the URL can be replaced with any date during O3, in the format YYYYMMDD.

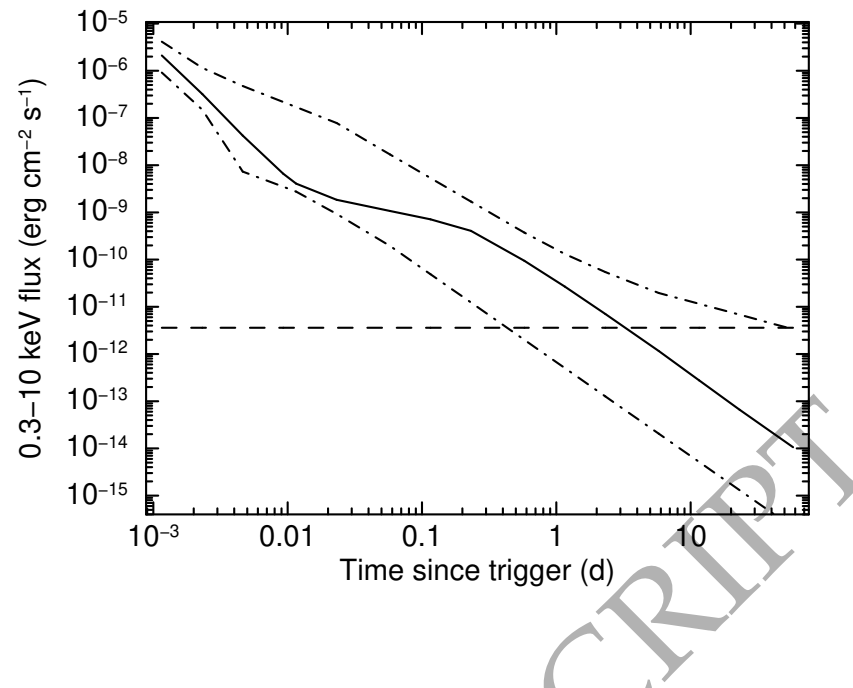

Figure 5. X-ray afterglow light-curve for on-axis short GRBs, scaled to $140 \mathrm{Mpc}$. The solid black line shows the median curve, while the dot-dashed lines mark the 25 th and 75 th percentiles. The grey horizontal dashed line indicates the average flux upper limit discussed in $\S 4.3$.

Table 4. X-ray upper limits (0.3-10 keV) for GW triggers where large-scale tiling patterns, were performed. The last column gives the approximate fraction of the (updated, if relevant) galaxyconvolved skymap observed by Swift.

\begin{tabular}{lccc} 
& & \\
\hline LVC trigger & $\begin{array}{c}3 \sigma \mathrm{UL}^{-1} \\
\left(\text { count s }^{-1}\right)\end{array}$ & $\begin{array}{c}\text { Obs. flux UL } \\
\left(\mathrm{erg} \mathrm{cm}^{-2} \mathrm{~s}^{-1}\right)\end{array}$ & $\begin{array}{c}\text { Fraction of } \\
\text { skymap covered }\end{array}$ \\
\hline S190412m & 0.087 & $3.7 \times 10^{-12}$ & 0.17 \\
S190425z & 0.15 & $6.5 \times 10^{-12}$ & 0.065 \\
S190426c & 0.046 & $2.0 \times 10^{-12}$ & 0.31 \\
S190510g & 0.091 & $3.9 \times 10^{-12}$ & 0.67 \\
S190718y & 0.025 & $1.1 \times 10^{-12}$ & 0.22 \\
S190728q & 0.070 & $3.0 \times 10^{-12}$ & 0.006 \\
S190814bv & 0.023 & $9.9 \times 10^{-13}$ & 0.90 \\
S190930t & 0.10 & $4.3 \times 10^{-12}$ & 0.03 \\
S191216ap & 0.19 & $8.2 \times 10^{-12}$ & 0.037 \\
S200114f & 0.092 & $4.0 \times 10^{-12}$ & 0.30 \\
S200115j & 0.021 & $9.0 \times 10^{-13}$ & 0.097 \\
S200224ca & 0.093 & $4.0 \times 10^{-12}$ & 0.80 \\
S200225q & 0.10 & $4.3 \times 10^{-12}$ & 0.51 \\
\hline
\end{tabular}

\section{FUTURE PROSPECTS}

A significant challenge for the Swift follow-up thus far has been the fact that more than half of the sources detected during O3 were Rank 3; that is, we cannot tell whether they are new sources, or just too faint to have been previously detected. As mentioned earlier, the ongoing SGWGS observations (75 per cent complete at the end of 2020 July) provide snapshot observations with which to compare later X-ray detections (something which is done automatically by the analysis software), which helps to mitigate the problem to some extent. More importantly, however, eROSITA (extended ROentgen Survey with an Imaging Telescope Array; 
Predehl 2017; Merloni, Nandra \& Predehl 2020), launched in 2019, will perform a new all-sky survey over the next few years, covering an energy range comparable to SwiftXRT. With expected soft $(0.5-2 \mathrm{keV})$ and hard $(2-10 \mathrm{keV})$ all-sky sensitivities of $\sim 4.4 \times 10^{-14}$ and $\sim 7.1 \times 10^{-13}$ erg $\mathrm{cm}^{-2} \mathrm{~s}^{-1}$ (for point sources), respectively, over the first six months, deepening to $\sim 1.1 \times 10^{-14}$ and $\sim 1.6 \times 10^{-13}$ erg $\mathrm{cm}^{-2} \mathrm{~s}^{-1}$ after the full four years (Predehl 2017), eROSITA will be able to detect, or place deeper upper limits on, many previously uncatalogued sources. More precisely, if we consider the Rank 3 sources identified by XRT throughout the $\mathrm{O} 3 \mathrm{run}$, and use a range of power-law indices $(\Gamma=0.5-2.0$, with a typical absorbing column of $\mathrm{N}_{\mathrm{H}}=3 \times 10^{20} \mathrm{~cm}^{-2}$ ) to estimate the corresponding fluxes, the eROSITA four year sensitivity should allow the survey to detect all of these sources over one or both energy bands. The sources detected by XRT would thus be classed as Rank 4 (catalogued) instead, if they were at a consistent or fainter flux than the earlier eROSITA detection; if brighter by at least $3 \sigma$, the source would be promoted to Rank 2 and marked for additional follow-up. This would decrease the number of candidate counterparts by about 95 per cent, significantly improving our ability to highlight the potentially interesting sources ${ }^{15}$.

We note that analysis by Basu-Zych et al. (2020) suggests that, while the eROSITA survey will significantly increase the number of X-ray detected normal (inactive) galaxies, this will still only be a few per cent of the total population (considering galaxies at a distance of 50-200 Mpc).

Besides eROSITA, Einstein Probe (Yuan et al. 2018), aimed for launch by the end of 2022 , has a large FOV of 3600 $\operatorname{deg}^{2}(\sim 1 \mathrm{sr})$, and will observe the whole sky over $0.5-5 \mathrm{keV}$ at high cadence, detecting X-ray transients with which Swift detections in GW follow-up observations can be compared. The ECLAIRs coded-mask detector onboard SVOM (Spacebased multi-band astronomical Variable Objects Monitor; Yu et al. 2020), due to be launched in 2021, has a 2 sr FOV with an energy bandpass down to $4 \mathrm{keV}$, and is expected to detect $\sim 70$ GRBs per year, adding to the chance that a short GRB coincident with a GW trigger will be detected.

As the sensitivity of the GW network improves, with more triggers at ever increasing distances, the incompleteness of galaxy catalogues will become more of a complication. Unless additional sensitive interferometers are included in the network, the positional errors will still remain large. However, a fourth LIGO interferometer in India is planned for the future, and KAGRA sensitivity should improve over the next few years. Selecting which triggers to follow, and optimising galaxy catalogues (which is currently being performed by a number of different groups in different ways), will be key to maximising the probability of detecting an EM counterpart.

\section{DATA AVAILABILITY}

The data underlying this article are available in the Swift archives at https://www.swift.ac.uk/swift_live/,

15 We note that the proprietary period for the German eROSITA data will be two years.
https://heasarc.gsfc.nasa.gov/cgi-bin/W3Browse/swift.pl and https://www.ssdc.asi.it/mmia/index.php?mission=swiftmastr, with the relevant target IDs provided at https://www.swift.ac.uk/GW/.

\section{ACKNOWLEDGEMENTS}

KLP, PAE, APB, AAB, NPMK, JPO, CP and MJP acknowledge funding from the UK Space Agency. NJK acknowledges support from NASA Grant 80NSSC19K0408. $\mathrm{AD}$ acknowledges financial contribution from the agreement ASI-INAF n.2017-14-H.0, while EA, MGB, SC, GC, AD, $\mathrm{AM}$ and GT acknowledge funding from the Italian Space Agency, contract ASI/INAF n. I/004/11/4. This work is also partially supported by a grant from the Italian Ministry of Foreign Affairs and International Cooperation Nr. MAE0065741 (AD), and by the Ministry of Education, Culture, Sports, Science, and Technology (MEXT) KAKENHI Grant Numbers 17H06357 and 17H06362 (TS). DBM acknowledges research grant 19054 from VILLUM FONDEN.

This work made use of data supplied by the UK Swift Science Data Centre at the University of Leicester. We thank Dr Gavin Lamb for useful discussions.

\section{REFERENCES}

Aartsen M.G. et a1., 2017, JInst, 12, P03012

Abbott B.P. et al., 2004, PhRvD, 69, 122001

Abbott B.P. et al., 2016a, ApJ, 826, L13

Abbott B.P. et al., 2016b, Phys.Rev.Lett., 116, 241103

Abbott B.P. et al., 2016c, Technical Report LIGO-P1600088

Abbott B.P. et al., 2017, ApJ, 848, L12

Abbott B.P. et al., 2019a, Phys.Rev.X, 9, 031040

Abbott B.P. et al., 2019b, ApJ, 886, 75

Abbott B.P. et al., 2020a, ApJ, 892, L3

Abbott R. et al., 2020b, Phys. Rev. D., 102, 043015

Abbott R. et al., 2020c, ApJ, 896, L44

Abbott R. et al., 2020d, Phys. Rev. Lett., 125, 101102

Acernese F et al., 2015, Class. Quantum Grav., 32, 024001

Ackley K. et al., 2020, A\&A, in press (arXiv:2002.01950)

Andreoni I., De K., Kasliwal M., Hiang Y., Liu X., 2020a, GCN Circ. 27075

Andreoni I. et al., 2020b, ApJ, 890, 131

Andreoni I., Goldstein D., Dobie D., Kasliwal M.M., 2019a, GCN Circ. 25488

Andreoni I. et al., 2019b, GCN Circ. 26424

Antier S. et al., 2020, MNRAS, 497, 5518

Barcons X. et al., 2007, A\&A, 476, 1191

Barr P., Mushotzky R.F., 1986, Nature, 320, 421

Barthelmy S.D. et al., 2005, Space Sci. Rev., 120, 143

Barthelmy S.D. et al., 2019a, GCN Circ. 24075

Barthelmy S.D. et al., 2019b, GCN Circ. 24255

Barthelmy S.D. et al., 2019c, GCN Circ. 24543

Barthelmy S.D. et al., 2019d, GCN Circ. 24646

Barthelmy S.D. et al., 2019e, GCN Circ. 25025

Barthelmy S.D. et al., 2019f, GCN Circ. 25174

Barthelmy S.D. et al., 2019g, GCN Circ. 25532

Barthelmy S.D. et al., 2019g, GCN Circ. 25718

Barthelmy S.D. et al., 2019i, GCN Circ. 25888

Barthelmy S.D. et al., 2019j, GCN Circ. 26234

Barthelmy S.D. et al., 2019k, GCN Circ. 26410

Barthelmy S.D. et al., 2020a, GCN Circ. 26649

Barthelmy S.D. et al., 2020b, GCN Circ. 26921 
Barthelmy S.D. et al., 2020c, GCN Circ. 27216

Barthelmy S.D. et al., 2020d, GCN Circ. 27369

Basu-Zych A.R. et al., 2020, MNRAS, in press (arXiv:2008.01870)

Berger E., 2014, ARA\&A, 52, 43

Bilicki M., Jarrett T.H., Peacock J.A., Cluver M.E., Steward L., 2014, ApJS, 210, 9

Boller T., Brandt W.N., Fink H., 1996, A\&A, 305, 53

Breeveld A.A. et al., 2019a, GCN Circ. 24296

Breeveld A.A. et al., 2019b, GCN Circ. 25525

Breeveld A.A. et al., 2020, GCN Circ. 27288

Buckley D.A.H., Jha S.W., Cooke J., Mogotsi M., 2019, GCN Circ. 24205

Burrows D.N. et al., 2005, Space Sci. Rev., 120, 165

Caccianiga A. et al., 2008, A\&A, 477, 735

Connaughton V. et al., 2016, ApJ, 826, L6

Connaughton V. et al., 2017, GCN Circ. 21506

Connaughton V. et al., 2018, ApJ, 853, L9

Corral A. et al., 2014, A\&A, 569, A71

Coulter D.A. et al., 2017, Sci, 358, 1556

Countryman S et al., 2020, GCN Circ. 27121

D'Avanzo P. et al., 2014, MNRAS, 442, 2342

Dobie D. et al., 2019, ApJ, 887, L13

Eichler D., Livio M., Piran T., Schramm D.N., 1989, Nature, 340, 126

Evans P.A., 2020, GCN Circ. 26777

Evans P.A., et al., 2012, ApJS, 203, 28

Evans P.A. et al., 2014, ApJS, 210, 8

Evans P.A. et al., 2016a, MNRAS, 455, 1522

Evans P.A. et al., 2016b, MNRAS, 460, L40

Evans P.A. et al., 2016c, MNRAS, 462, 1591

Evans P.A. et al., 2017, Science, 358, 1565

Evans P.A. et al., 2019a, GCN Circ. 24273

Evans P.A. et al., 2019b, GCN Circ. 24541

Evans P.A. et al., 2019c, GCN Circ. 25151

Evans P.A. et al., 2019d, GCN Circ. 25400

Evans P.A. et al., 2019e, GCN Circ. 25545

Evans P.A. et al., 2019f, GCN Circ. 25966

Evans P.A. et al., 2019g, GCN Circ. 26238

Evans P.A. et al., 2019h, GCN Circ. 26475

Evans P.A. et al., 2019i, GCN Circ. 26498

Evans P.A. et al., 2020a, GCN Circ. 26787

Evans P.A. et al., 2020b, GCN Circ. 26791

Evans P.A. et al., 2020c, GCN Circ. 26798

Evans P.A. et al., 2020d, GCN Circ. 26855

Evans P.A. et al., 2020e, GCN Circ. 27524

Evans P.A. et al., 2020f, GCN Circ. 27526

Evans P.A. et al., 2020g, ApJS, 247, 54

Gehrels N. et al., 2004, ApJ, 611, 1005

Giommi P. et al., 2019, A\&A, 631, A116

Goldstein A. et al., 2017, ApJ, 848, L14

Gompertz B.P. et al., 2020, MNRAS, 497, 726

Graham M.J. et al., 2020, Phys. Rev. Let, 124, 251102

Greiner J., Burgess J.M., Savchenko V. Yu H.-F., 2016, ApJ, 827, L38

Hallinan G. et al., 2017, Sci, 358, 1579

HAWC Collaboration, 2019, GĆN Circ. 26472

IceCube Collaboration, 2019a, GCN Circ. 25192

IceCube Collaboration, 2019b, GCN Circ. 25197

IceCube Collaboration, 2019c, GCN Circ. 25210

IceCube Collaboration, 2019d, GCN Circ. 26460

IceCube Collaboration, 2019e, GCN Circ. 26463

IceCube Collaboration, 2020, GCN Circ. 27043

Jaodand, A.et al., 2019, GCN Circ. 25822

KAGRA Collaboration, 2019, NatAs, 3, 35

Kamble A., Kaplan D.L.A., 2013, Int. J. Mod. Phys. D, 22, 1341011

Kasliwal M.M. et al., 2019a, GCN Circ. 24191

Kasliwal M.M. et al., 2019b, GCN Circ. 25199
Kasliwal M.M. et al., 2019c, GCN Circ. 25207

Kasliwal M.M. et al., 2020a, GCN Circ. 27051

Kasliwal M.M. et al., 2020b, submitted to ApJ (arXiv:2006.11306)

Klingler N.J. et al., 2019, ApJSS, 245, 15

Kuin N.P.M. et al., 2019a, GCN Circ. 24767

Kuin N.P.M. et al., 2019b, GCN Circ. 24861

Kuin N.P.M. et al., 2019c, GCN Circ. 24863

Jencson J., De K., Anand S., Kasliwal M.M., Andreoni I., Ahumada T., Perley D.A., 2019, GCN Circ. 24233

Laha S., Gronwall C., Klinger N.J., Lien A.Y., Page K.L., Tohuvavohu A., 2020, GCN Circ. 27234

Li L.-X., Paczyǹski, B., 1998, ApJ, 507, L59

Lien A.Y. et al., 2019a, GCN Circ. 24114

Lien A.Y. et al., 2019b, GCN Circ. 24391

Lien A.Y. et al., 2019c, GCN Circ. 24582

Lien A.Y. et al., 2019d, GCN Circ. 24725

Lien A.Y. et al., 2019e, GCN Circ. 25044

Lien A.Y. et al., 2019f, GCN Circ. 25200

Lien A.Y. et al., 2019g, GCN Circ. 25533

Lien A.Y. et al., 2019h, GCN Circ. 25533

Lien A.Y. et al., 2019i, GCN Circ. 25889

Lien A.Y. et al., 2019j, GCN Circ. 26313

Lien A.Y. et al., 2019k, GCN Circ. 26453

Lien A.Y. et al., 2020a, GCN Circ. 26723

Lien A.Y. et al., 2020b, GCN Circ. 26940

Lien A.Y. et al., 2020c, GCN Circ. 27401

LIGO Scientific Collaboration, 2015, Class. Quantum Grav., 32, 074001

LIGO Scientific Collaboration, Virgo Collaboration, 2019a, GCN Circ. 24069

LIGO Scientific Collaboration, Virgo Collaboration, 2019b, GCN Circ. 24098

LIGO Scientific Collaboration, Virgo Collaboration, 2019c, GCN Circ. 24109

LIGO Scientific Collaboration, Virgo Collaboration, 2019d, GCN Circ. 24141

LIGO Scientific Collaboration, Virgo Collaboration, 2019e, GCN Circ. 24158

LFGO Scientific Collaboration, Virgo Collaboration, 2019f, GCN Circ. 24168

LIGO Scientific Collaboration, Virgo Collaboration, 2019g, GCN Circ. 24228

LIGO Scientific Collaboration, Virgo Collaboration, 2019h, GCN Circ. 24237

LIGO Scientific Collaboration, Virgo Collaboration, 2019i, GCN Circ. 24277

LIGO Scientific Collaboration, Virgo Collaboration, 2019j, GCN Circ. 24279

LIGO Scientific Collaboration, Virgo Collaboration, 2019k, GCN Circ. 24375

LIGO Scientific Collaboration, Virgo Collaboration, 20191, GCN Circ. 24377

LIGO Scientific Collaboration, Virgo Collaboration, 2019m, GCN Circ. 24411

LIGO Scientific Collaboration, Virgo Collaboration, 2019n, GCN Circ. 24442

LIGO Scientific Collaboration, Virgo Collaboration, 2019o, GCN Circ. 24448

LIGO Scientific Collaboration, Virgo Collaboration, 2019p, GCN Circ. 24462

LIGO Scientific Collaboration, Virgo Collaboration, 2019q, GCN Circ. 24489

LIGO Scientific Collaboration, Virgo Collaboration, 2019r, GCN Circ. 24503

LIGO Scientific Collaboration, Virgo Collaboration, 2019s, GCN Circ. 24522 
LIGO Scientific Collaboration, Virgo Collaboration, 2019t, GCN Circ. 24570

LIGO Scientific Collaboration, Virgo Collaboration, 2019u, GCN Circ. 24584

LIGO Scientific Collaboration, Virgo Collaboration, 2019v, GCN Circ. 24591

LIGO Scientific Collaboration, Virgo Collaboration, 2019w, GCN Circ. 24598

LIGO Scientific Collaboration, Virgo Collaboration, 2019x, GCN Circ. 24621

LIGO Scientific Collaboration, Virgo Collaboration, 2019y, GCN Circ. 24632

LIGO Scientific Collaboration, Virgo Collaboration, 2019z, GCN Circ. 24640

LIGO Scientific Collaboration, Virgo Collaboration, 2019aa, GCN Circ. 24656

LIGO Scientific Collaboration, Virgo Collaboration, 2019ab, GCN Circ. 24717

LIGO Scientific Collaboration, Virgo Collaboration, 2019ac, GCN Circ. 24922

LIGO Scientific Collaboration, Virgo Collaboration, 2019ad, GCN Circ. 24950

LIGO Scientific Collaboration, Virgo Collaboration, 2019ae, GCN Circ. 24987

LIGO Scientific Collaboration, Virgo Collaboration, 2019af, GCN Circ. 24998

LIGO Scientific Collaboration, Virgo Collaboration, 2019ag, GCN Circ. 25012

LIGO Scientific Collaboration, Virgo Collaboration, 2019ah, GCN Circ. 25048

LIGO Scientific Collaboration, Virgo Collaboration, 2019ai, GCN Circ. 25049

LIGO Scientific Collaboration, Virgo Collaboration, 2019aj, GCN Circ. 25087

LIGO Scientific Collaboration, Virgo Collaboration, 2019ak, GCN Circ. 25094

LIGO Scientific Collaboration, Virgo Collaboration, 2019al, GCN Circ. 25107

LIGO Scientific Collaboration, Virgo Collaboration, 2019am, GCN Circ. 25115

LIGO Scientific Collaboration, Virgo Collaboration, 2019an, GCN Circ. 25138

LIGO Scientific Collaboration, Virgo Collaboration, 2019ao, GCN Circ. 25164

LIGO Scientific Collaboration, Virgo Collaboration, 2019ap, GCN Circ. 25187

LIGO Scientific Collaboration, Virgo Collaboration, 2019aq, GCN Circ. 25208

LIGO Scientific Collaboration, Virgo Collaboration, 2019ar, GCN Circ. 25249

LIGO Scientific Collaboration, Virgo Collaboration, 2019as, GCN Circ. 25296

LIGO Scientific Collaboration, Virgo Collaboration, 2019at, GCN Circ. 25301

LIGO Scientific Collaboration, Virgo Collaboration, 2019au, GCN Circ. 25324

LIGO Scientific Collaboration, Virgo Collaboration, 2019av, GCN Circ. 25333

LIGO Scientific Collaboration, Virgo Collaboration, 2019aw, GCN Circ. 25367

LIGO Scientific Collaboration, Virgo Collaboration, 2019ax, GCN Circ. 25442

LIGO Scientific Collaboration, Virgo Collaboration, 2019ay, GCN Circ. 25497

LIGO Scientific Collaboration, Virgo Collaboration, 2019az, GCN Circ. 25503

LIGO Scientific Collaboration, Virgo Collaboration, 2019ba, GCN Circ. 25504
LIGO Scientific Collaboration, Virgo Collaboration, 2019bb, GCN Circ. 25549

LIGO Scientific Collaboration, Virgo Collaboration, 2019bc, GCN Circ. 25554

LIGO Scientific Collaboration, Virgo Collaboration, 2019bd, GCN Circ. 25606

LIGO Scientific Collaboration, Virgo Collaboration, 2019be, GCN Circ. 25614

LIGO Scientific Collaboration, Virgo Collaboration, 2019bf, GCN Circ. 25695

LIGO Scientific Collaboration, Virgo Collaboration, 2019bg, GCN Circ. 25707

LIGO Scientific Collaboration, Virgo Collaboration, 2019bh, GCN Circ. 25723

LIGO Scientific Collaboration, Virgo Collaboration, 2019bi, GCN Circ. 25753

LIGO Scientific Collaboration, Virgo Collaboration, 2019bj, GCN Circ. 25773

LIGO Scientific Collaboration, Virgo Collaboration, 2019bk, GCN Circ. 25778

LIGO Scientific Collaboration, Virgo Collaboration, 2019bl, GCN Circ. 25782

LIGO Scientific Collaboration, Virgo Collaboration, 2019bm, GCN Circ. 25814

LIGO Scientific Collaboration, Virgo Collaboration, 2019bn, GCN Circ. 25829

LIGO Scientific Collaboration, Virgo Collaboration, 2019bo, GCN Circ. 25861

LIGO Scientific Collaboration, Virgo Collaboration, 2019bp, GCN Circ. 25871

LIGO Scientific Collaboration, Virgo Collaboration, 2019bq, GCN Circ. 25876

LIGO Scientific Collaboration, Virgo Collaboration, 2019br, GCN Circ. 25883

LIGO Seientific Cóllaboration, Virgo Collaboration, 2019bs, GCN Circ. 25909

LIGO Scientific Collaboration, Virgo Collaboration, 2019bt, GCN Girc. 25968

LIGO Scientific Collaboration, Virgo Collaboration, 2019bu, GCN Circ. 26182

LIGO Scientific Collaboration, Virgo Collaboration, 2019bv, GCN Circ. 26202

LIGO Scientific Collaboration, Virgo Collaboration, 2019bw, GCN Circ. 26218

LIGO Scientific Collaboration, Virgo Collaboration, 2019bx, GCN Circ. 26222

LIGO Scientific Collaboration, Virgo Collaboration, 2019by, GCN Circ. 26245

LIGO Scientific Collaboration, Virgo Collaboration, 2019bz, GCN Circ. 26250

LIGO Scientific Collaboration, Virgo Collaboration, 2019ca, GCN Circ. 26254

LIGO Scientific Collaboration, Virgo Collaboration, 2019cb, GCN Circ. 26263

LIGO Scientific Collaboration, Virgo Collaboration, 2019cc, GCN Circ. 26265

LIGO Scientific Collaboration, Virgo Collaboration, 2019cd, GCN Circ. 26288

LIGO Scientific Collaboration, Virgo Collaboration, 2019ce, GCN Circ. 26303

LIGO Scientific Collaboration, Virgo Collaboration, 2019cf, GCN Circ. 26334

LIGO Scientific Collaboration, Virgo Collaboration, $2019 \mathrm{cg}$, GCN Circ. 26350

LIGO Scientific Collaboration, Virgo Collaboration, 2019ch, GCN Circ. 26383

LIGO Scientific Collaboration, Virgo Collaboration, 2019ci, GCN Circ. 26395 
LIGO Scientific Collaboration, Virgo Collaboration, 2019cj, GCN Circ. 26402

LIGO Scientific Collaboration, Virgo Collaboration, 2019ck, GCN Circ. 26413

LIGO Scientific Collaboration, Virgo Collaboration, 2019cl, GCN Circ. 26417

LIGO Scientific Collaboration, Virgo Collaboration, $2019 \mathrm{~cm}$, GCN Circ. 26441

LIGO Scientific Collaboration, Virgo Collaboration, 2019cn, GCN Circ. 26454

LIGO Scientific Collaboration, Virgo Collaboration, 2019co, GCN Circ. 26505

LIGO Scientific Collaboration, Virgo Collaboration, 2019cp, GCN Circ. 26513

LIGO Scientific Collaboration, Virgo Collaboration, 2019cq, GCN Circ. 26518

LIGO Scientific Collaboration, Virgo Collaboration, 2019cr, GCN Circ. 26543

LIGO Scientific Collaboration, Virgo Collaboration, 2019cs, GCN Circ. 26570

LIGO Scientific Collaboration, Virgo Collaboration, 2019ct, GCN Circ. 26572

LIGO Scientific Collaboration, Virgo Collaboration, 2019cu, GCN Circ. 26585

LIGO Scientific Collaboration, Virgo Collaboration, 2020a, GCN Circ. 26640

LIGO Scientific Collaboration, Virgo Collaboration, 2020b, GCN Circ. 26641

LIGO Scientific Collaboration, Virgo Collaboration, 2020c, GCN Circ. 26642

LIGO Scientific Collaboration, Virgo Collaboration, 2020d, GCN Circ. 26657

LIGO Scientific Collaboration, Virgo Collaboration, 2020e, GCN Circ. 26665

LIGO Scientific Collaboration, Virgo Collaboration, 2020f, GCN Circ. 26675

LIGO Scientific Collaboration, Virgo Collaboration, 2020g, GCN Circ. 26688

LIGO Scientific Collaboration, Virgo Collaboration, 2020h, GCN Circ. 26715

LIGO Scientific Collaboration, Virgo Collaboration, 2020i, GCN Circ. 26734

LIGO Scientific Collaboration, Virgo Collaboration, 2020j, GCN Circ. 26759

LIGO Scientific Collaboration, Virgo Collaboration, $2020 \mathrm{k}$, GCN Circ. 26785

LIGO Scientific Collaboration, Virgo Collaboration, 20201, GCN Circ. 26807

LIGO Scientific Collaboration, Virgo Collaboration, 2020m, GCN Circ. 26906

LIGO Scientific Collaboration, Virgo Collaboration, 2020n, GCN Circ. 26926

LIGO Scientific Collaboration, Virgo Collaboration, 2020o, GCN Circ. 27014

LIGO Scientific Collaboration, Virgo Collaboration, 2020p, GCN Circ. 27036

LIGO Scientific Collaboration, Virgo Collaboration, 2020q, GCN Circ. 27042

LIGO Scientific Collaboration, Virgo Collaboration, 2020r, GCN Circ. 27092

LIGO Scientific Collaboration, Virgo Collaboration, 2020s, GCN Circ. 27096

LIGO Scientific Collaboration, Virgo Collaboration, 2020t, GCN Circ. 27130

LIGO Scientific Collaboration, Virgo Collaboration, 2020u, GCN Circ. 27184

LIGO Scientific Collaboration, Virgo Collaboration, 2020v, GCN Circ. 27193
LIGO Scientific Collaboration, Virgo Collaboration, 2020w, GCN Circ. 27214

LIGO Scientific Collaboration, Virgo Collaboration, 2020x, GCN Circ. 27229

LIGO Scientific Collaboration, Virgo Collaboration, 2020y, GCN Circ. 27262

LIGO Scientific Collaboration, Virgo Collaboration, 2020z, GCN Circ. 27278

LIGO Scientific Collaboration, Virgo Collaboration, 2020aa, GCN Circ. 27292

LIGO Scientific Collaboration, Virgo Collaboration, 2020ab, GCN Circ. 27306

LIGO Scientific Collaboration, Virgo Collaboration, 2020ac, GCN Circ. 27347

LIGO Scientific Collaboration, Virgo Collaboration, 2020ad, GCN Circ. 27358

LIGO Scientific Collaboration, Virgo Collaboration, 2020ae, GCN Circ. 27382

LIGO Scientific Collaboration, Virgo Collaboration, 2020af, GCN Circ. 27388

LIGO Scientific Collaboration, Virgo Collaboration, 2020ag, GCN Circ. 27419

Magee M., Maguire K., Torres M., Jonker P., Fraser M., 2019, GCN Circ. 25209

Mateos S. et al., 2008, A\&A, 492, 51

McBrien O. et al., 2019, GCN Circ. 26485

McHardy I., 1985, Space Sci. Rev., 40, 559

Merloni A., Nandra, K., Predehl, P., 2020, NatAs, 4, 634

Metzger B.D., 2019, LRR, 23, 1

Metzger B.D. et al., 2010, MNRAS, 406, 2650

Mochkovitch R., Hernanz M., Isern J., Martin X., 1993, Nature, 361,236

Mushotzky R.F., Done C., Pounds K.A., 1993, ARA\&A, 31, 717

Nousek J.A. et al., 2006, ApJ, 642, 389

Oates S.R. et al. 2019a, GCN Circ. 26471

Oates S.R. et al., 2019b, GCN Circ. 26501

Oates S.R. et al., 2019c, GCN Circ. 25901

Oates S.R. et al., 2019d, GCN Circ. 25939

Oates S.R. et al., 2019e, GCN Circ. 25984

Oates S.R. et al., 2019f, GCN Circ. 26471

Oates S.R. et al., 2019g, GCN Circ. 26501

Oates S.R. et al., 2020a, GCN Circ. 26808

Oates S.R. et al., 2020b, GCN Circ. 27153

Oates S.R. et al., 2020c, GCN Circ. 27400

Oh K. et al., 2018, ApJS, 23, 4

Palmer D.M. et al., 2019a, GCN Circ. 24158

Palmer D.M. et al., 2019b, GCN Circ. 24454

Palmer D.M. et al., 2019c, GCN Circ. 24610

Palmer D.M. et al., 2019d, GCN Circ. 24938

Palmer D.M. et al., 2019e, GCN Circ. 25096

Palmer D.M. et al., 2019f, GCN Circ. 25341

Palmer D.M. et al., 2019g, GCN Circ. 25617

Palmer D.M. et al., 2019h, GCN Circ. 25843

Palmer D.M. et al., 2019i, GCN Circ. 26192

Palmer D.M. et al., 2019j, GCN Circ. 26348

Palmer D.M. et al., 2019k, GCN Circ. 26466

Palmer D.M. et al., 2020a, GCN Circ. 26748

Palmer D.M. et al., 2020b, GCN Circ. 27023

Palmer D.M. et al., 2020c, GCN Circ. 27217

Perley D., Copperwheat C.M., Taggart K.L., 2019a, GCN Circ. 24204

Perley D. et al., 2019b, GCN Circ. 24331

Pian E. et al., 2017, Nat, 551, 67

Predehl P., 2017, AN, 338, 159

Reusch S., Stein R., Perley D., Anand S., 2020, GCN Circ. 27068

Roming P.W.A. et al., 2005, Space Sci. Rev., 120, 95

Sakamoto T. et al., 2019a, GCN Circ. 24184

Sakamoto T. et al., 2019b, GCN Circ. 24518 
Sakamoto T. et al., 2019c, GCN Circ. 24645

Sakamoto T. et al., 2019d, GCN Circ. 24966

Sakamoto T. et al., 2019e, GCN Circ. 25127

Sakamoto T. et al., 2019f, GCN Circ. 25704

Sakamoto T. et al., 2019g, GCN Circ. 25846

Sakamoto T. et al., 2019h, GCN Circ. 26211

Sakamoto T. et al., 2019i, GCN Circ. 26365

Sakamoto T. et al., 2019j, GCN Circ. 26557

Sakamoto T. et al., 2020a, GCN Circ. 26779

Sakamoto T. et al., 2020b, GCN Circ. 27058

Sakamoto T. et al., 2020c, GCN Circ. 27147

Sakamoto T. et al., 2020d, GCN Circ. 27289

Savchenko V. et al., 2017, ApJ, 848, L15

Saxton R.D., Read A.M., Esquej P., Freyberg M.J., Altieri B., Bermejo D., 2008, A\&A, 480, 611

Shanks T., Georgantopoulos I., Stewart G.C., Pounds K.A., Boyle B.J., Griffiths R.E., 1991, Nature, 353, 315

Singer L. et al., 2019, GCN Circ. 26479

Singer L. et al., 2016, ApJ, 829, L15

Smartt S.J. et al., 2019, GCN Circ. 25204

Smith K.W. et al., 2019, GCN Circ. 24210

Springer R.W., HAWC Collaboration, 2016, NPPP, 279, 87

Stein R. et al., 2019a, GCN Circ. 25899

Stein R., Reusch S., Perley, D., Andreoni I., Coughlin M., 2019b, GCN Circ. 25899

Stewart A., Dobie D., Murphy T., Lenc E., Wang Z., Kaplan D., Hotan A., Moss V., 2019, GCN Circ. 25487

Thakur A.L. et al., 2020, MNRAS, submitted (arXiv:2007.04998)

Tohuvavohu A., 2018, J. Phys.: Conf. Ser., 1085, 032010

Tohuvavohu A., Kennea, J.A., Swift Gravitational Wave Followup Group, 2018, in González, G., Hynes R., eds., Proc. of the IAU, vol. 13 (S338), 'Gravitational Wave Astrophysics: Early Results from Gravitational Wave Searches and Electromagnetic Counterparts', 53

Tohuvavohu A., Evans P., Siegel M., Marshall F., Cenko B., Kennea J., 2019a, GCN Circ. 24305

Tohuvavohu A. et al., 2019b, GCN Circ. 24353

Tohuvavohu A. et al., 2019c, GCN Circ. 25220

Tohuvavohu A. et al., 2019d, GCN Circ. 25964

Tohuvavohu A., Kennea, J.A., DeLaunay, J., Palmer, D.M., Cenko S.B., Barthelmy S., 2020, ApJ, 900, 35

Troja E. et al., 2017, Nat, 551, 71

Valeev A.F. et al., 2019, GCN Circ. 26591

White D.J., Daw E.J., Dhillon V.S., 2011, CQGra, 28, 085016

Wyatt S.D., Tohuvavohu A., Arcavi I., Lundquist M.J., Howell D.A., Sand D.J., 2020, ApJ, 894, 127

Yu S.-j., Gonzalez F., Wei J.-y., Zhang S.-n., Cordier B., 2020, ChA\&A, 44, 269

Yuan W. et al., 2018, SPIE, 10699, 25

\section{APPENDIX A: O3 TRIGGERS}

Table A1 lists relevant information for all the LVC triggers from the third observing run, including those which were subsequently retracted, noting which were followed-up by Swift. 


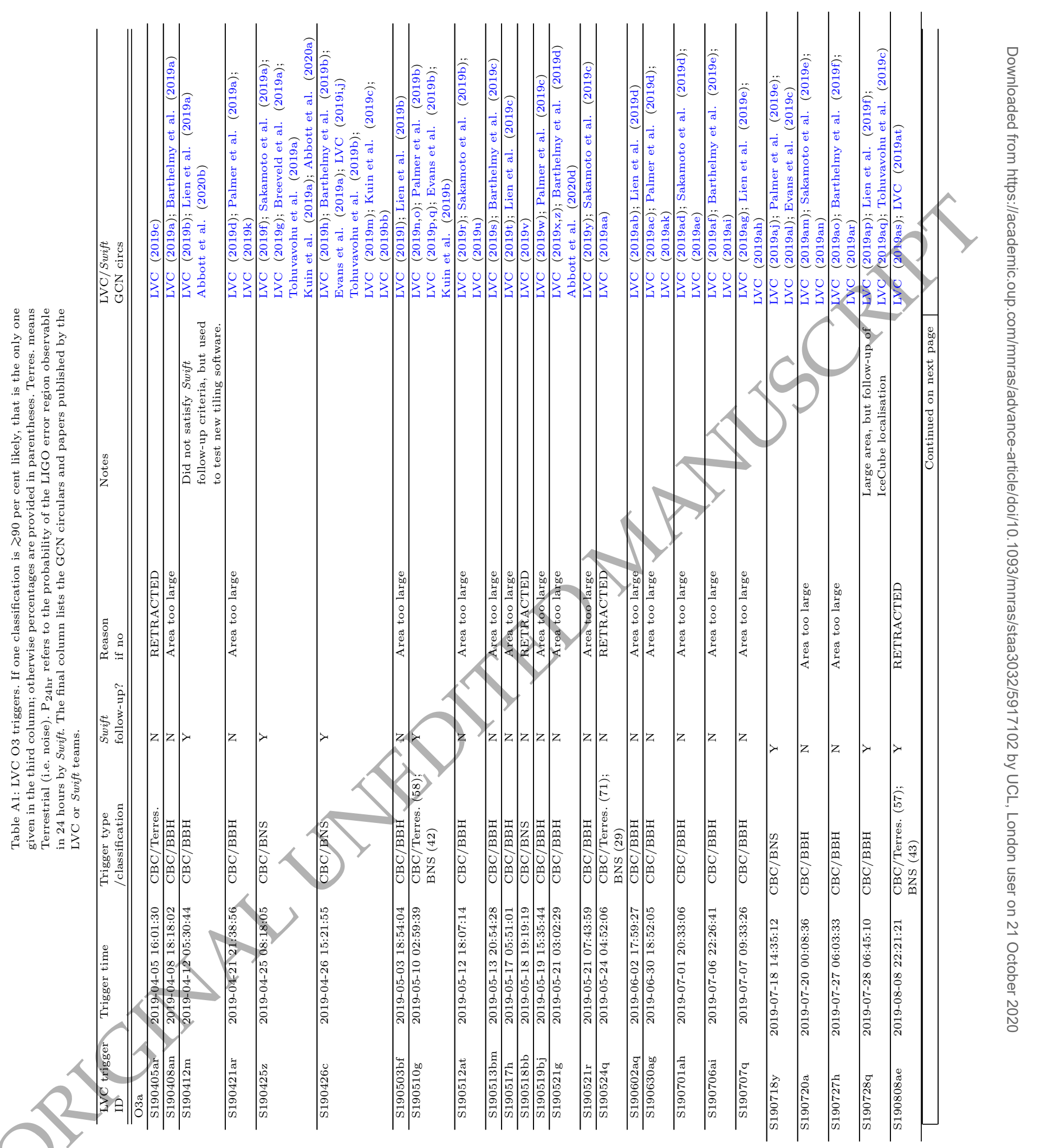




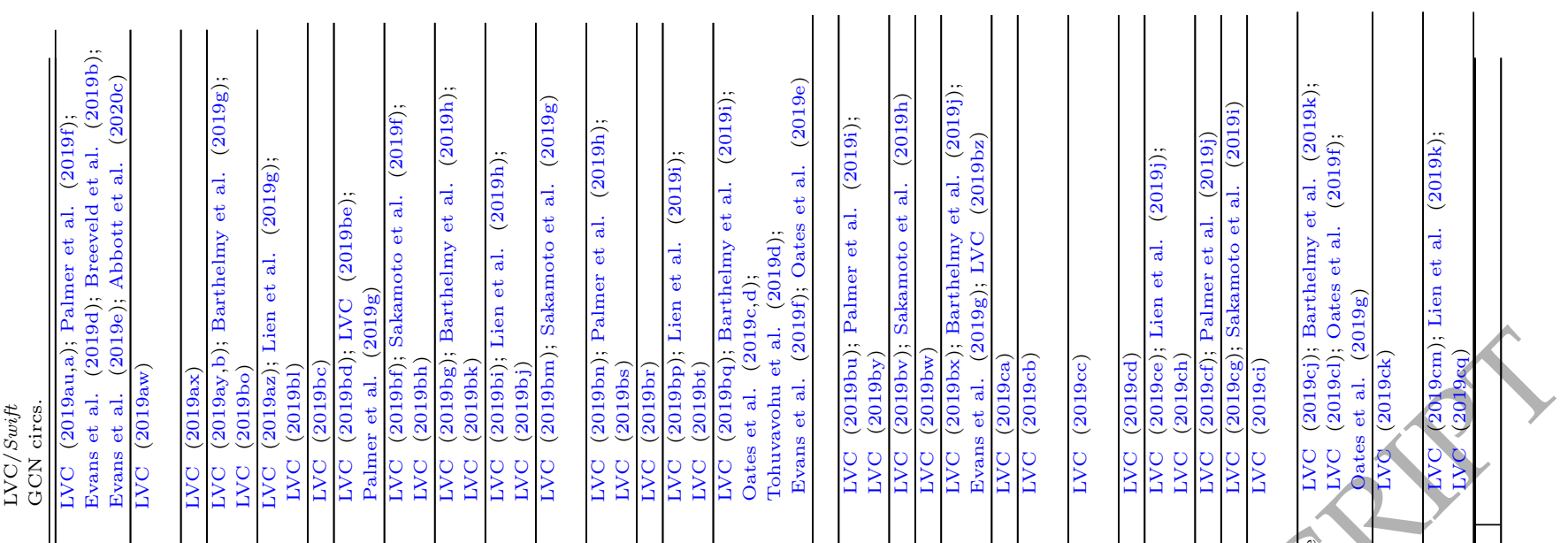

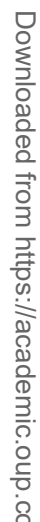

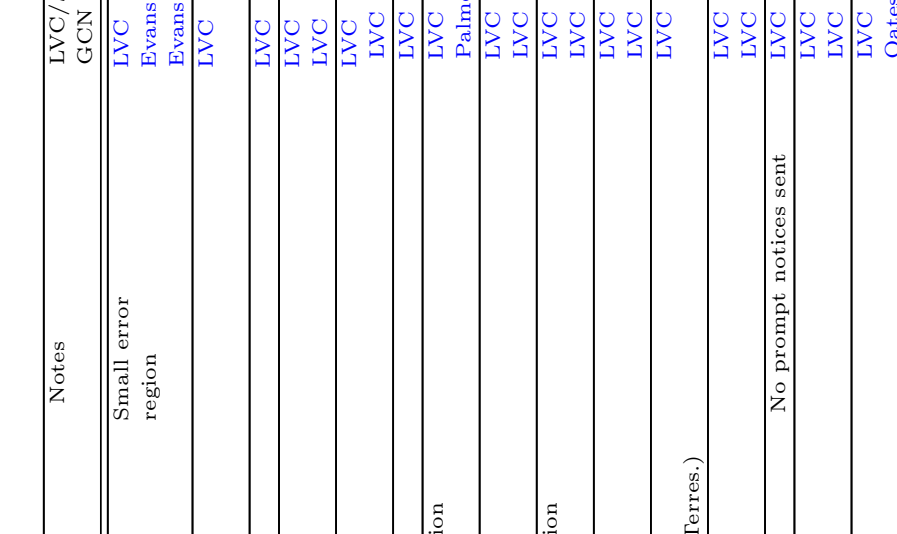

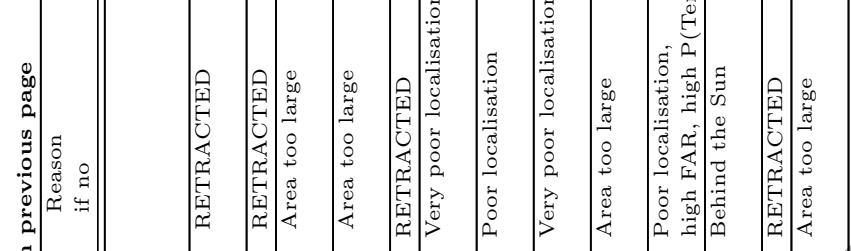

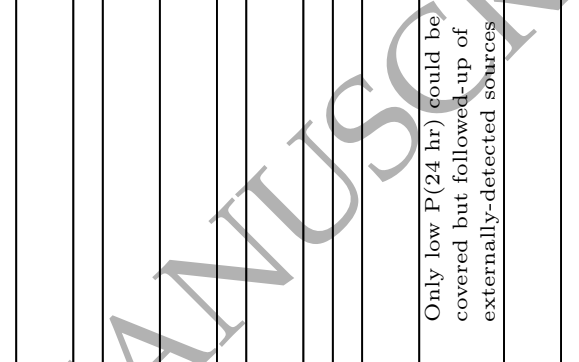

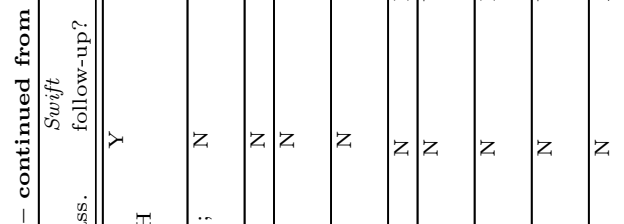

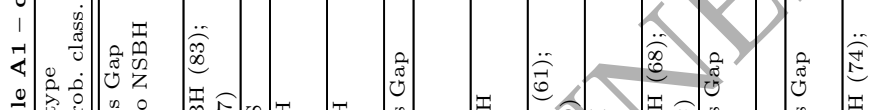

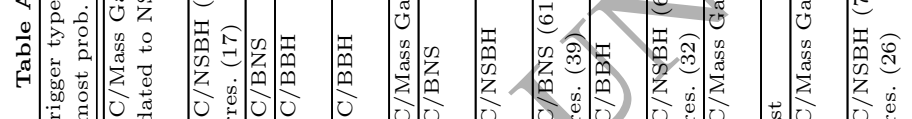

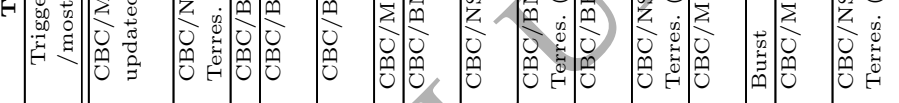

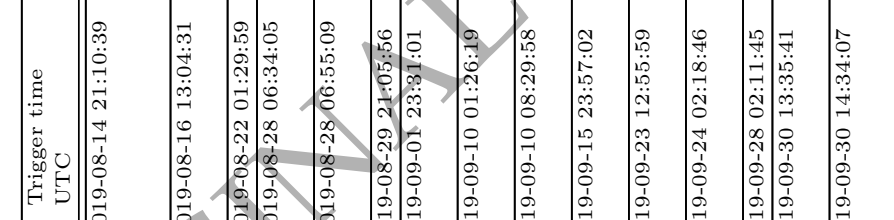

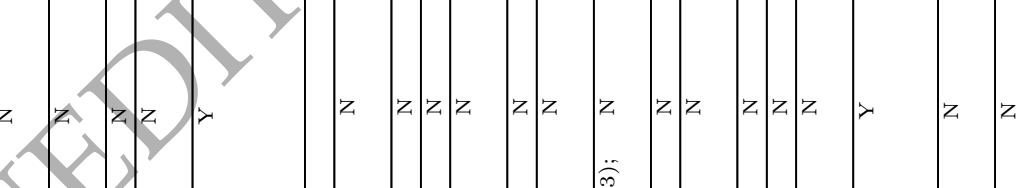

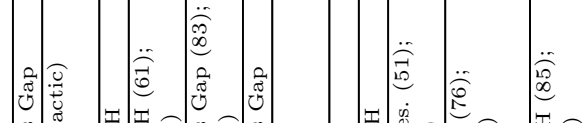

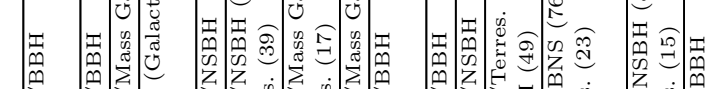

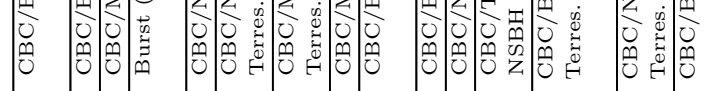

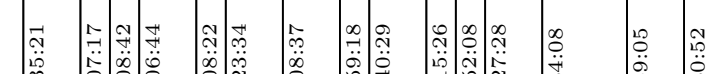

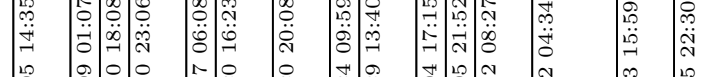

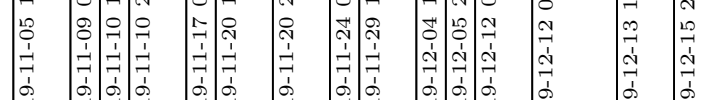

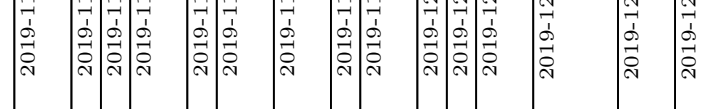

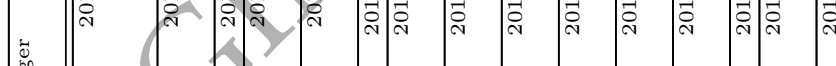

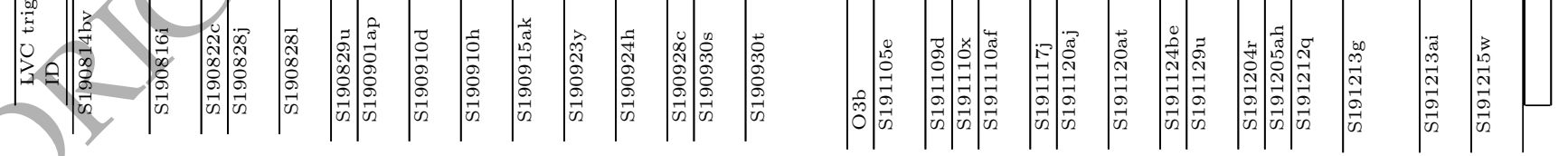


Table A1 - continued from previous page

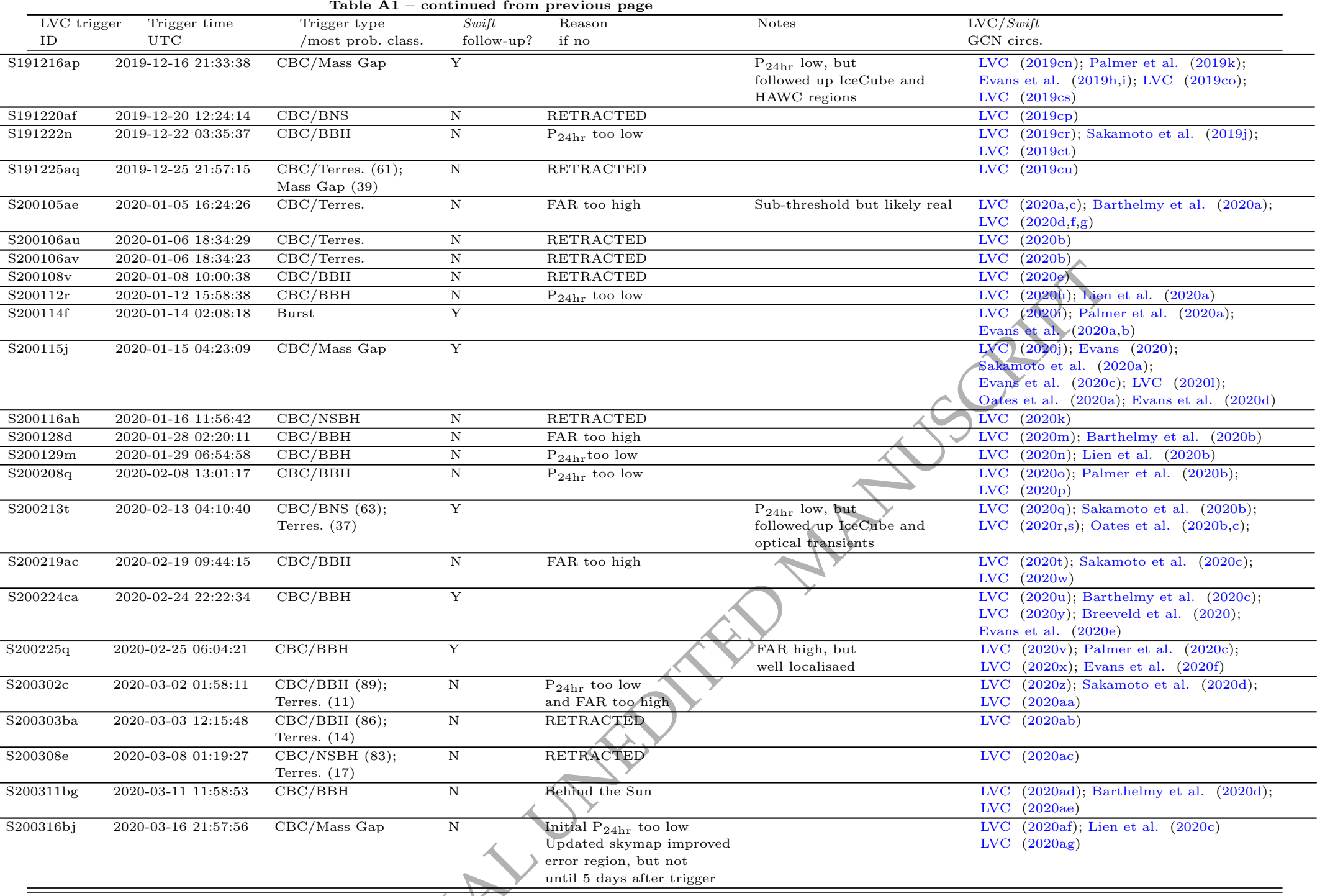

